\title{
RASS-SDSS galaxy cluster survey
}

\section{Scaling relations of galaxy clusters}

\author{
P. Popesso ${ }^{1}$, A. Biviano ${ }^{2}$, H. Böhringer ${ }^{1}$, M. Romaniello ${ }^{3}$, and W. Voges ${ }^{1}$ \\ 1 Max-Planck-Institut fur extraterrestrische Physik, 85748 Garching, Germany \\ e-mail: popesso@mpe.mpg.de \\ 2 INAF - Osservatorio Astronomico di Trieste, via G. B. Tiepolo 11, 34131 Trieste, Italy \\ 3 European Southern Observatory, Karl Scharzschildstr. 2, Garching b. München, Germany
}

Received 27 August 2004 / Accepted 16 November 2004

\begin{abstract}
We use the RASS-SDSS galaxy cluster sample to compare the quality of optical and X-ray luminosities as predictors of other cluster properties such as their masses, temperatures, and velocity dispersions. We use the SDSS spectroscopic data to estimate the velocity dispersions and the virial masses of a subsample of 69 clusters within $r_{500}$ and $r_{200}$. The ASCA temperature of the intra-cluster medium, $T_{\mathrm{X}}$, is retrieved from the literature for a subsample of 49 clusters. For this subsample we estimate the cluster masses also by using the mass-temperature relation. We show that the optical luminosity, $L_{\mathrm{op}}$, correlates with the cluster mass much better than the X-ray luminosity, $L_{\mathrm{X}} . L_{\mathrm{op}}$ can be used to estimate the cluster mass with an accuracy of $40 \%$ while $L_{\mathrm{X}}$ can predict the mass only with a $55 \%$ accuracy. We show that correcting $L_{\mathrm{X}}$ for the effect of a cool core at the center of a cluster lowers the scatter of the $L_{\mathrm{X}}-M$ relation only by $3 \%$. We find that the scatter observed in the $L_{\mathrm{op}}-L_{\mathrm{X}}$ relation is determined by the scatter of the $L_{\mathrm{X}}-M$ relation. The mass-to-light ratio in the SDSS $i$ band clearly increases with the cluster mass with a slope $0.2 \pm 0.08$. The optical and X-ray luminosities correlate in an excellent way with both $T_{\mathrm{X}}$ and $\sigma_{V}$ with an orthogonal scatter of $20 \%$ in both relations. Moreover, $L_{\mathrm{op}}$ and $L_{\mathrm{X}}$ can predict variables with the same accuracy both. We conclude that the cluster optical luminosity is a key cluster parameter since it can give important information about fundamental cluster properties such as the mass, the velocity dispersion, and the temperature of the intra-cluster medium.
\end{abstract}

Key words. X-rays: galaxies: clusters - surveys

\section{Introduction}

Clusters of galaxies are the most massive gravitationally bound systems in the universe. The mass is the most important property of these systems. The cluster mass function and its evolution provide constraints on the evolution of large-scale structure and important cosmological parameters such as $\Omega_{\mathrm{m}}$ and $\sigma_{8}$. The mass-to-light ratio of clusters provides one of the most robust determinations of $\Omega_{\mathrm{m}}$ in connection with the observed light density in the Universe via the Oort (1958) method. For these reasons, over the last 70 years (starting with Zwicky 1933, 1937; and Smith 1936), much effort has been spent on measuring the mass of clusters using a number of techniques. These include: (i) dynamical methods applied to the galaxy distributions derived from redshift surveys; or (ii) X-ray observations of the distribution and temperature of the diffuse hot gas in the intracluster medium (ICM); (iii) gravitational lensing; and (iv) observations of the Sunyaev-Zeldovich effect. All these methods are in general quite expensive in terms of the observational resources required, especially for high redshift clusters.
In the literature several comparisons have been made between the different methods for determining the mass of the clusters. These include comparisons between the X-ray and strong-lensing methods (see, e.g., Wu 2000) in cores of clusters, between X-ray and weak lensing methods (see, e.g., Smail et al. 1997), and between the dynamical and the X-ray methods (see, e.g., Wu 2000). In particular, Girardi et al. (1998) and Rines et al. (2003) have shown that consistent results can be obtained from these last two methods.

The existence of a fundamental plane for the global properties of galaxy clusters (Schaeffer et al. 1993; Adami et al. 1998; Fujita \& Takahara 1999) naturally implies that other properties, such as cluster luminosities, velocity dispersions, $\mathrm{X}$-ray temperatures, can be used to infer the cluster masses. It is not known which correlates better with the cluster mass, the X-ray or the optical luminosity. Reiprich \& Böhringer (2002, R02 hereafter) showed that a tight correlation exists between the X-ray total luminosity of the clusters and the mass, with a scatter of $60 \%$. Girardi et al. $(2000,2002)$ analysed the relation between mass and optical luminosity in the blue band, and detetrmined the mass-to-light ratio for a sample of 162 clusters using inhomogeneous photometric data. 
Yee \& Ellingson (2003) analysed a sample of 16 X-ray luminous clusters from the Canadian Network for Observational Cosmology (CNOC) survey. They used the cluster optical richness, rather than the optical luminosity, and showed that it is well correlated with other global properties of galaxy clusters as their velocity dispersion such as their intracluster gas temperature, and their total mass. Lin et al. (2003) analysed a sample of 27 clusters with near-infrared data from the Two Micron All Sky Survey (2MASS) and available X-ray temperature. They showed that the $K$-band luminosity of a cluster can be used to estimate its mass with $45 \%$ accuracy.

Analysing the relation between optical luminosity and cluster mass is not an easy task. The lack of optical wide field surveys in the past did not allow measuring in the proper way the optical luminosity in galaxy systems. Until now the uncertainties in luminosity determination came from the corrections for the calibration of inhomogenous photometric data, background galaxy contamination and the need to extrapolate the sum of measured luminosities of galaxy members to include faint galaxies and the outer parts of the systems, beyond the region studied. The use of the Sloan Digital Sky Survey (SDSS) for the optical data allows us to overcome all the problems related to the optical luminosity estimation.

In this paper we use the RASS-SDSS galaxy cluster sample (Popesso et al. 2004) to study the correlations between the optical luminosity and other important properties of galaxy clusters such as their mass, line-of-sight velocity dispersion, temperature and X-ray luminosity. Moreover, the RASS-SDSS galaxy cluster sample allows us to compare the correlations of the optical and the X-ray luminosity with other global properties of the systems. The excellence of the second release of the SDSS (SDSS-DR2, Abazajian et al. 2004) in terms of its size, depth and sky coverage, the accurate photometry in 5 different optical wavebands, and the detailed spectroscopy for more than 260000 galaxies give us unprecedented advantages in comparison to the previous studies. Firstly, the sky coverage (3324 $\mathrm{deg}^{2}$ ) gives us the possibility of studying a large sample of clusters with completely homogeneous photometric data. Secondly, the accurate estimation of the spectroscopic redshift for a large subsample of galaxies allows us to define an accurate membership for any cluster and, thus, perform a detailed dynamical analysis of the system within the same survey. Thirdly, the sky coverage of the survey also makes it possible to overcome the well known problem of the statistical subtraction of the galaxy background. We use large areas of the survey to define a mean global galaxy background, and a region close to the clusters to determine the local galaxy background in order to check for systematics in the field subtraction. Finally, the magnitude limit of the SDSS DR2 in all the five bands is sufficiently deep (e.g. $r_{\text {lim }}=22.2,95 \%$ completeness) that, at the mean redshift of our cluster sample $(z \sim 0.10)$, the cluster luminosity function (LF) is sampled down to a significant part of the faint end. Moreover, the use of the ROSAT All Sky Survey (RASS) allows us to define also the X-ray properties in a homogeneous way for all the systems and perform a detailed comparison with the optical luminosity.

In this paper we show that the optical luminosity, $L_{\mathrm{op}}$, is strongly correlated with the other global properties of a galaxy cluster, allowing its use as an estimator for quantities such as the velocity dispersion, the mass, and the intra-cluster gas temperature, $T_{\mathrm{X}}$. We demonstrate that $L_{\mathrm{op}}$ is a better predictor of the virial mass than the X-ray luminosity, which makes $L_{\mathrm{op}}$ an important defining parameter of galaxy clusters, and an extremely useful cosmological tool. Throughout this paper we use $H_{0}=70 \mathrm{~km} \mathrm{~s}^{-1} \mathrm{Mpc}^{-1}$ in a flat cosmology with $\Omega_{0}=0.3$ and $\Omega_{\Lambda}=0.7$ (e.g. Tegmark et al. 2004).

\section{The cluster sample and the data}

The ROSAT-SDSS galaxy cluster catalog comprises 130 systems detected in the ROSAT All Sky Survey (RASS). The $\mathrm{X}$-ray cluster properties and the redshifts have been taken from different catalogs of X-ray selected clusters: the ROSATESO flux limited X-ray cluster sample (REFLEX, Böhringer et al. 2001, 2002), the Northern ROSAT All-sky cluster sample (NORAS, Böhringer et al. 2000), the NORAS 2 cluster sample (Retzlaff 2001), the ASCA Cluster Catalog (ACC) from Horner et al. (2001) and the Group Sample (GS) of Mulchaey et al. (2003).

The optical photometric data are taken from the SDSS DR2 (Fukugita 1996; Gunn et al. 1998; Lupton et al. 1999; York et al. 2000; Hogg et al. 2001; Eisenstein et al. 2001; Smith et al. 2002; Strauss et al. 2002; Stoughton et al. 2002; Blanton et al. 2003; Abazajian et al. 2003). The SDSS consists of an imaging survey of $\pi$ steradians of the northern sky in the five passbands $u, g, r, i, z$, in the entire optical range from the atmospheric ultraviolet cutoff in the blue to the sensitivity limit of silicon in the red. The survey is carried out using a $2.5 \mathrm{~m}$ telescope, an imaging mosaic camera with $30 \mathrm{CCDs}$, two fiberfed spectrographs and a $0.5 \mathrm{~m}$ telescope for the photometric calibration. The imaging survey is taken in drift-scan mode. The imaging data are processed with a photometric pipeline (PHOTO, Lupton et al. 2001) specially written for the SDSS data. For each cluster we defined a photometric galaxy catalog as described in Sect. 3 of Popesso et al. (2004; see also Yasuda et al. 2001).

For the analysis in this paper we use only SDSS Model magnitudes. Because of a bug in PHOTO, found during the completion of DR1, the model magnitudes were systematically under-estimated by about $0.2-0.3 \mathrm{mag}$ for galaxies brighter than 20th magnitude, and accordingly the measured radii were systematically too large. This problem has been fixed in the SDSS DR2, therefore the model magnitude can be considered a good estimate of the galaxy total luminosity at any magnitude and is not dependent on the seeing, like the Petrosian magnitudes.

The spectroscopic component of the survey is carried out using two fiber-fed double spectrographs, covering the wavelength range 3800-9200 $\AA$, over 4098 pixels. They have a resolution $\Delta \lambda / \lambda$ varying between 1850 and 2200, and together they are fed by 640 fibers, each with an entrance diameter of 3 arcsec. The fibers are manually plugged into plates inserted into the focal plane; the mapping of fibers to plates is carried out by a tiling algorithm (Blanton et al. 2003) that optimizes observing efficiency in the presence of large-scale structure. The finite diameter of the fiber cladding prevents fibers on any 
given plate from being placed less than 55 arcsec apart. For any given plate, a series of fifteen-minute exposures is carried out until the mean signal to noise ratio $(\mathrm{S} / \mathrm{N})$ per resolution element exceeds 4 for objects with fiber magnitudes (i.e., as measured through the 3 aperture of the fiber) brighter than $g=20.2$ and $i=19.9$, as determined by preliminary reductions done at the observing site. Under good conditions (dark, clear skies and good seeing), this typically requires a total of $45 \mathrm{~min}$ of exposure.

To create a homogeneous catalog of X-ray cluster properties, we have computed the X-ray luminosity using only RASS data for all clusters in the sample. The X-ray luminosity has been calculated with the growth curve analysis (GCA) method used for the NORAS and REFLEX cluster surveys (Böhringer et al. 2000) based on the RASS3 data base (Voges et al. 1999). The GCA method is optimized for the detection of the extended emission of clusters by assessing the plateau of the background subtracted cumulative count rate curve. We use as final result the total flux inside the radius $r_{200}$ which is corrected for the missing flux estimated via the assumption of a standard $\beta$-model for the X-ray surface brightness (see Böhringer et al. 2000 for more details). The correction is typically only $8-10 \%$ illustrating the high effectiveness of the GCA method for sampling the flux of extended sources. For a subsample of 49 galaxy clusters we have also compiled from the literature the ASCA temperature of the ICM.

\section{The cluster optical luminosity}

The total optical luminosity of a cluster has to be computed after the subtraction of the foreground and background galaxy contamination. We consider two different approaches to the statistical subtraction of the galaxy background. We compute the local background number counts in an annulus around the cluster and a global background number counts from the mean of the magnitude number counts determined in five different SDSS sky regions, each with an area of $30 \mathrm{deg}^{2}$. In our analysis we show the results obtained using the optical luminosity estimated with the second method. The optical luminosity is then computed following the prescription of Popesso et al. (2004). The reader is referred to that paper for a detailed discussion about the comparison between optical luminosities calculated with different methods.

\section{Cluster members selection and mass estimation}

To select the members of each system and estimate the mass we use the redshifts in the SDSS spectroscopic sample. The SDSS spectroscopic pipeline (spectro1d) assigns a final redshift to each object spectrum by choosing the emission or cross-correlation redshift with the highest confidence level. The emission-redshift is obtained by matching the list of candidate emission lines against a list of common galaxy and quasar emission lines. The cross-correlated redshift is estimated by cross-correlating the spectrum with stellar, emissionline galaxy, and quasar template spectra.

In order to select the cluster members, we proceed in two steps. In the first step we follow the method of
Girardi et al. (1993). Namely, we select only galaxies within a circle of one Abell radius $(2.15 \mathrm{Mpc})$, and eliminate those with redshift $\left|c z-c z_{\text {cluster }}\right|>4000 \mathrm{~km} \mathrm{~s}^{-1}$, where $z_{\text {cluster }}$ is the mean cluster redshift as given in the X-ray catalogues (see Sect. 2). We then define the weighted gaps (see also Beers et al. 1990) in the $z$-distribution of the remaining galaxies, and reject galaxies separated from the main cluster body by a weighted gap $\geq 4$. This allows us to define the cluster limits in velocity space.

In the second step of our procedure for membership selection, we consider all galaxies (not only those within an Abell radius) with a velocity within the limits defined with the gapper procedure, and apply the method of Katgert et al. (2004) to these galaxies. The method takes into account both the velocities and the clustercentric positions of the galaxies (we take the X-ray center as the dynamical center of the cluster). The method is identical to that of den Hartog \& Katgert (1996) when the cluster sample contains at least 45 galaxies, and it is a simplified version of it for smaller samples (for more details, see Appendix A in Katgert et al. 2004).

The virial analysis (see, e.g., Girardi et al. 1998) is then performed on the clusters with at least 10 member galaxies. The velocity dispersion is computed using the biweight estimator (Beers et al. 1990). The virial masses are corrected for the surface pressure term (The \& White 1986) by adopting a profile of Navarro et al. (1996, 1997; NFW) with concentration parameter $c=4$ (this profile has been found to describe the average mass profile of rich clusters by Katgert et al. 2004). Correction for the surface term requires knowledge of the $r_{200}$ radius, for which we adopt Carlberg et al.'s (1997a) definition (see Eq. (8) in that paper), as a first guess. After the virial mass has been corrected for the surface pressure term, we refine our $r_{200}$ estimate using the virial mass density itself. Let $M_{\text {vir }}$ be the virial mass (corrected for the surface term) contained in a volume of radius equal to the clustercentric distance of the most distant cluster member in the sample, i.e. the aperture radius $r_{\text {ap }}$. The radius $r_{200}$ is then given by:

$r_{200} \equiv r_{\mathrm{ap}}\left[\rho_{\mathrm{vir}} /\left(200 \rho_{\mathrm{c}}\right)\right]^{1 / 2.4}$

where $\rho_{\text {vir }} \equiv 3 M_{\text {vir }} /\left(4 \pi r_{\text {ap }}^{3}\right)$ and $\rho_{\mathrm{c}}(z)$ is the critical density at redshift $z$ in the adopted cosmology. The exponent in Eq. (1) is the one that describes the average cluster mass density profile near $r_{200}$, as estimated by Katgert et al. (2004) for an ensemble of 59 rich clusters. Similarly, $r_{500}$ is estimated by setting 500 instead of 200 in Eq. (1). Finally, a $c=4$ NFW profile is used to interpolate (or, in a few cases, extrapolate) the virial

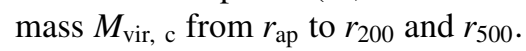

Our clusters span a wide range in mass; since clusters of different masses have different concentrations (see, e.g. Dolag et al. 2004) we should in principle compute the cluster masses, $M$, using a different concentration parameter $c$ for each cluster. According to Dolag et al. (2004), $c \propto M^{-0.102}$. Taking $c=4$ for clusters as massive as those analysed by Katgert et al. (2004), $M \simeq 2 \times 10^{15} M_{\odot}$, Dolag et al.'s scaling implies that our clusters span a range $c \simeq 3-6$. Using $c=6$ instead of $c=4$ makes the mass estimates $4 \%$ and $10 \%$ higher at, respectively, $r_{200}$ and $r_{500}$, while using $c=3$ makes the mass estimates lower by the same factors. This effect clearly being much smaller than 
the observational uncertainties, we assume the same $c=4$ in the analysis for all clusters.

Even if the completeness level of the SDSS spectroscopic sample is very high, in the central regions of galaxy clusters this level is likely to drop because fibers cannot be placed closer than 55 arcsec. We estimate that the spectroscopic completeness drops to $\sim 70 \%$ in the central $\sim 0.1 \mathrm{Mpc}$. This affects the observed number density profile of a cluster, and hence our estimate of the projected mean harmonic pairwise separation $\mathrm{s}\left\langle R_{i j}^{-1}\right\rangle$, and, as a consequence, also our virial mass estimates (see, e.g., Beers et al. 1984). Using the average cluster number density profile, and the relation between the core radius of this profile and $\left\langle R_{i j}^{-1}\right\rangle$, as given by Girardi et al. (1995, 1998), we estimate that this effect of incompleteness translates into an average over-estimate of the virial mass of only $\sim 5 \%$. Since the effect is very small, and much smaller than the observational uncertainties, we neglect this correcting factor in the following analysis.

There are several indications that cluster galaxies of later morphological and/or spectral type, have wider velocity distributions than early-type cluster galaxies (Moss \& Dickens 1977; Biviano et al. 2002, and references therein). As a consequence, we also estimate the virial masses by considering only cluster members along the red sequence in the $u-i$ vs. $i$ colour-magnitude diagram. When only the red-sequence cluster members are used to compute the virial masses, these masses are $\sim 25 \%$ smaller than when all the cluster members are used. A similar effect has been discussed by Biviano et al. (1997) for the galaxy clusters of the ESO Nearby Abell Cluster Survey, and by Carlberg et al. (1997b) for the galaxy clusters of the CNOC. The effect is generally interpreted as evidence for ongoing accretion of blue field galaxies onto the cluster, before complete virialization (see, e.g., the discussion in Biviano \& Katgert 2003). Since this effect is not negligible, in the following we consider both the mass estimates obtained using only the red-sequence galaxies, and the mass estimates obtained using all the cluster members. However, for the sake of conciseness, we only plot results for the mass estimates obtained using all the cluster members.

To check the consistency of our mass estimates with those obtained from X-ray data, we retrieve the latter from R02, for a subsample of 10 clusters with optical mass estimates. Figure 1 shows the overall agreement between the X-ray mass from R02 and the optical mass calculated in this work (empty squares). The figure shows also the comparison between optical mass and the mass obtained from the $M-T$ relation for 6 more clusters with know ASCA temperature but with unknown X-ray mass (filled squares). It is mainly for the systems with evident substructures in the redshift distribution (the empty and filled squares surrounded by big triangles in the figure) that the two mass estimates are in disagreement, as expected. In the following analysis we do not consider these clusters with strong optical substructures. We omit in Fig. 1 the comparison between the mass estimated from the $M-T_{\mathrm{X}}$ relation (see Eq. (2) below) and the direct measure of the X-ray mass (R02). They are in very good agreement since the $M-T_{\mathrm{X}}$ relation is derived from R02 data, and the scatter in the relation is very small as shown by the errors in Eq. (2) and Fig. 3.

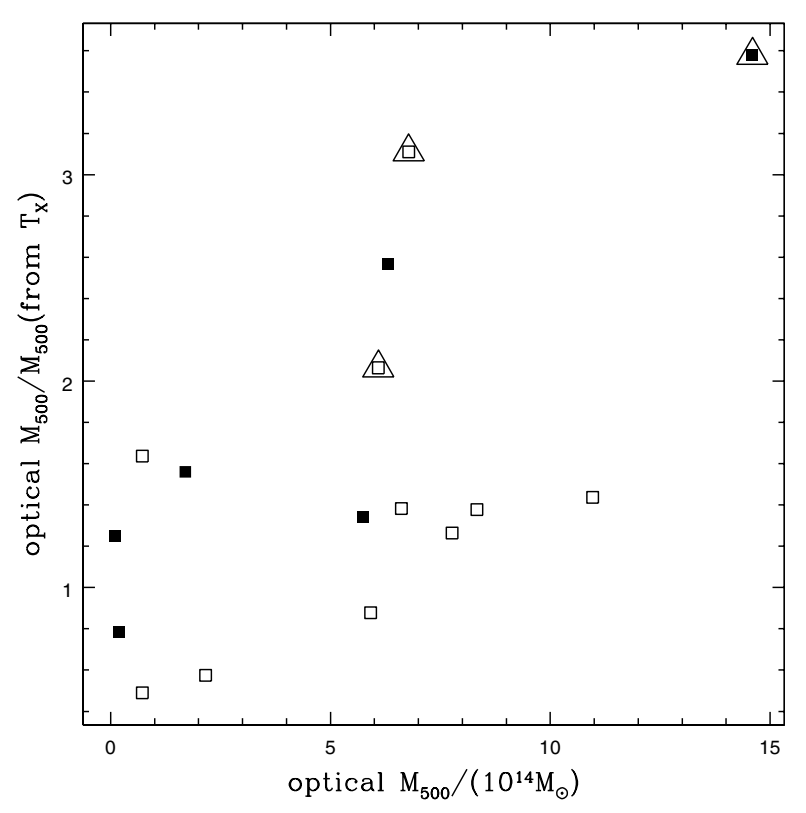

Fig. 1. Comparison of $M_{500}$ estimated from X-ray data with the mass estimated from the dynamical analysis of the optical data. We know the temperature for 16 clusters of the subsample with known optical mass. For 10 of them the measure of the X-ray mass is given by $\mathrm{R} 02$. We compare the optical mass with the X-ray mass of R02, when available, and with the mass derived from the $M-T_{\mathrm{X}}$ relation (Eq. (2)) in the other case. The empty squares are the ratio between the optical mass and the mass given by R02. The filled squares are the ratio between the optical mass and the mass derived from the $M-T_{\mathrm{X}}$ relation (Eq. (2)). The squares surrounded by big triangles are clusters with known optical substructures.

\subsection{Masses estimated from the $M-T_{\mathrm{X}}$ relation}

We use the $M-T_{\mathrm{X}}$ relation to estimate the mass for the subsample of 49 clusters with known ASCA temperature. First, we consider the $M-T_{\mathrm{X}}$ relations provided by Finoguenov et al. (2001, hereafter F01). F01 provided several $M-T_{\mathrm{X}}$ relations that had been estimated using different samples of systems: the cluster sample of R02 (HIFLUGCS + 60 more clusters) and a sample of 39 clusters with known temperature profile from ASCA data (Markevitch et al. 1998). We notice that the masses estimated with the $M-T_{\mathrm{X}}$ relation of F01 obtained from clusters with known temperature profile are systematically smaller by a factor 1.5-2 than both the virial masses obtained from the analysis of the galaxy distribution, and also the X-ray masses of R02. The F01 sample comprises only 9 of the 45 clusters included in the R02 sample with temperature higher than $5 \mathrm{keV}$. The masses of these 9 clusters are in good agreement with the masses estimated in R02 (which assumes the isothermality of the ICM) but, as shown in Fig. 2, the high-mass region is not well sampled. Moreover, the presence of 4 systems in the low mass regime with $\beta \sim 0.3$ (where $\beta$ is the exponent of the $\beta$-model used to calculate the X-ray mass, see F01 or R02 for more details) implies both a steepening of the $M-T_{\mathrm{X}}$ relation and a decrease of its normalization.

Therefore, instead of using the $M-T_{\mathrm{X}}$ relation of F01, we prefer to recalibrate the $M-T_{\mathrm{X}}$ relation using the data of R02 in order to obtain both the $M_{500}-T_{\mathrm{X}}$ and the $M_{200}-T_{\mathrm{X}}$ 


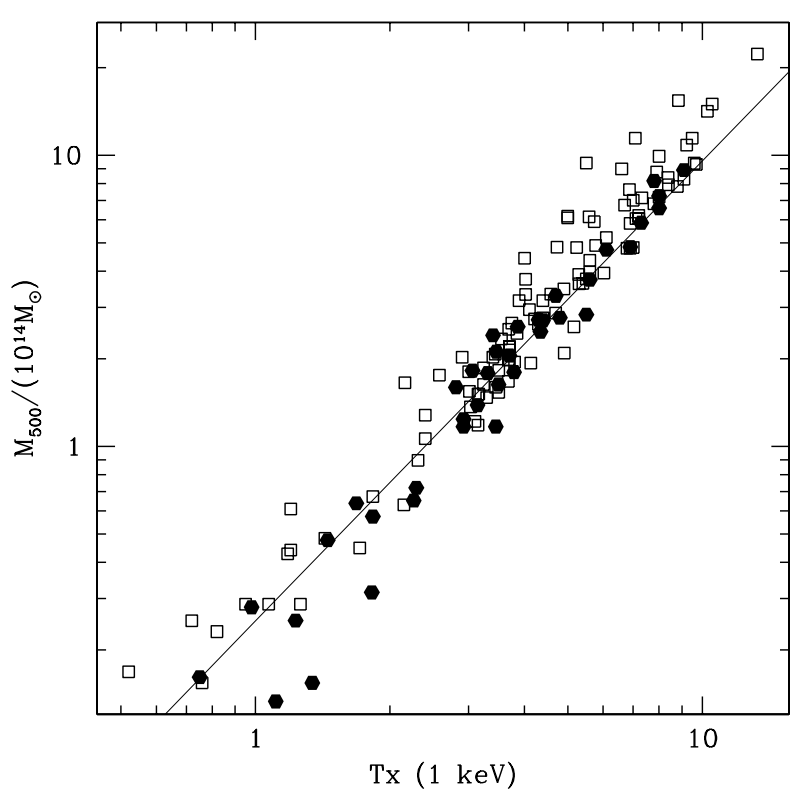

Fig. 2. Relation between mass and temperature. The mass is calculated using a $\beta$-model and the temperature of the ICM. The empty squares are the clusters of R02, while the filled hexagons are taken from F01. The solid line in the figure is the best fit line given by F01.

relations. The data are fitted with the ODRPACK routine (Akritas \& Bershady 1996) and the errors are calculated using a bootstrap method. In the following analysis the mass is calculated from the temperature with the following relations:

$M_{500}=2.89 \pm 0.29 \times 10^{13} T_{\mathrm{X}}^{1.59 \pm 0.04}$

$M_{200}=4.69 \pm 0.36 \times 10^{13} T_{\mathrm{X}}^{1.59 \pm 0.05}$.

The normalization of the $M-T_{\mathrm{X}}$ relation in Eq. (2) is $60 \%$ higher than that of the usual $M-T_{\mathrm{X}}$ relation of F01 estimated with the sample with known $T_{\mathrm{X}}$ profiles. There is much better agreement (within $1 \sigma$ ) with the relation of F01 estimated excluding from the sample the groups with $\beta$ lower than 0.4 . Figure 3 shows the best fit for the enlarged sample of R02 while Fig. 4 shows the comparison of our best fit with the relation predicted by the hydrodynamical simulations.

\section{Correlation of the optical and X-ray luminosities with the cluster global properties}

In this section we examine the correlation of the optical luminosity $L_{\mathrm{op}}$ and the X-ray luminosity $L_{\mathrm{X}}$ with quantities derived from the optical and X-ray data, such as the total mass, the velocity dispersion, the X-ray temperature, $r_{500}$, and $r_{200}$. The main motivation in deriving these dependences is to use $L_{\mathrm{op}}$ and $L_{\mathrm{X}}$, as predictors of the other quantities. Moreover, we will compare the quality of the two quantities $L_{\mathrm{op}}$ and $L_{\mathrm{X}}$ as predictors. To quantify all the dependences, a linear regression in $\log$-log space is performed using a numerical orthogonal distance regression method (ODRPACK). The fits are performed using the form

$\log \left(L_{\mathrm{op}} /\left(10^{12} L_{\odot}\right)\right)=\alpha \times \log \left(P_{\mathrm{C}}\right)+\beta$

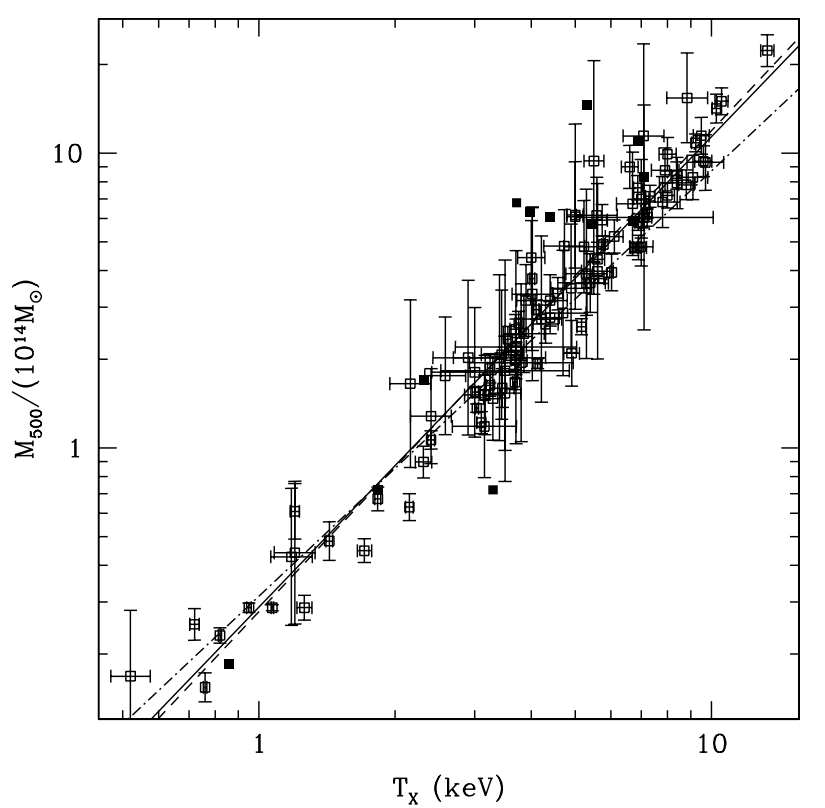

Fig. 3. Mass-temperature relation calibrated with the cluster mass and temperature used in R02. The new normalization in the $M-T_{\mathrm{X}}$ relation is $60 \%$ higher than in the classical $M-T_{\mathrm{X}}$ relation of F01. The solid line is the best fit line obtained using the enlarged sample of R02. The dotted line is the best fit line obtained fitting the clusters with $T_{\mathrm{X}}$ higher than $4.5 \mathrm{keV}$, while the dashed-dotted line is the best fit line derived from the fit of systems with temperature lower than $4.5 \mathrm{keV}$.

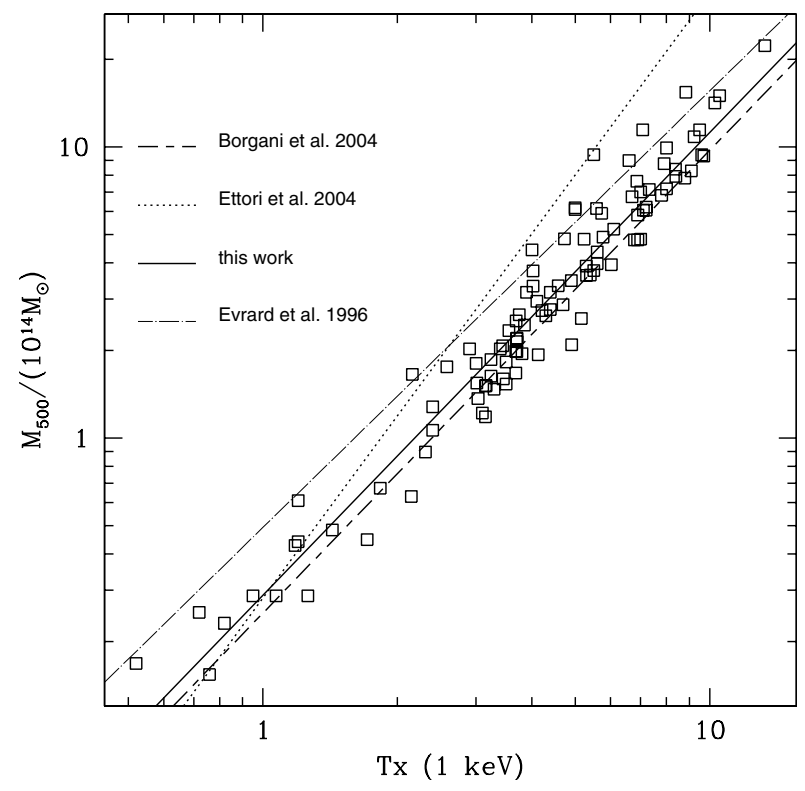

Fig. 4. Comparison of the $M-T_{\mathrm{X}}$ relation obtained with the sample from R02 and the theoretical prediction from Borgani et al. (2004), Ettori et al. (2004), Evrard et al. (1996).

$\log \left(L_{\mathrm{X}} /\left(10^{44} \mathrm{erg} \mathrm{s}^{-1}\right)\right)=\alpha \times \log \left(P_{\mathrm{C}}\right)+\beta$

where $P_{\mathrm{C}}$ is the cluster global property, and the errors of each variable are transformed into $\log$ space as $\Delta \log (x)=$ $\log (e)\left(x^{+}-x^{-}\right) /(2 x)$, where $x^{+}$and $x^{-}$denote the upper and lower boundary of the error range of the quantity, respectively. In all the correlations we analyse separately the sample with masses derived from the dynamical analysis (69 clusters) and 


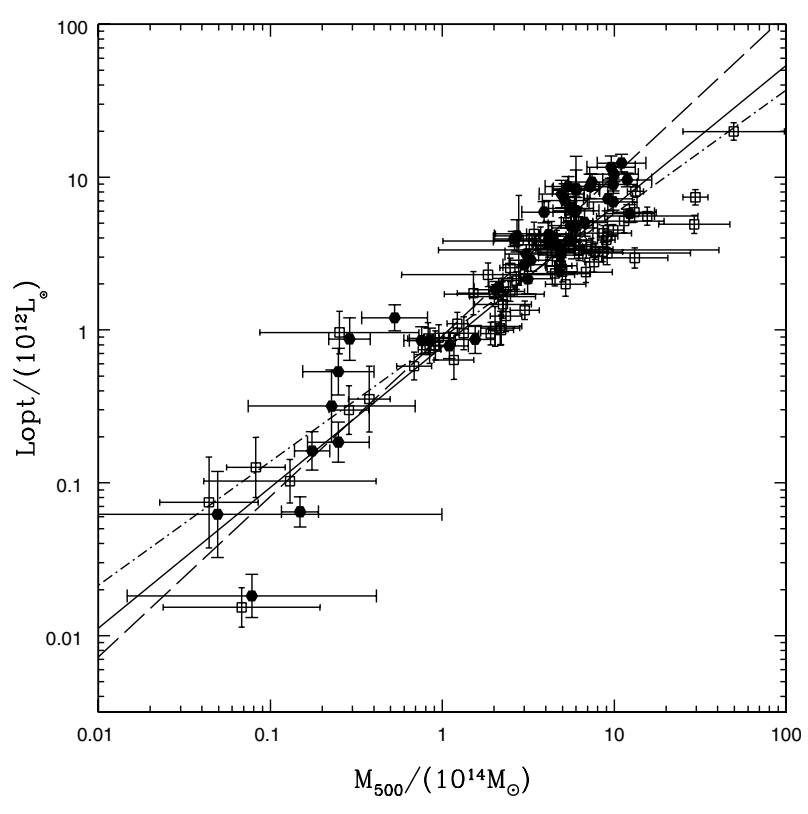

Fig. 5. $L_{\mathrm{op}}-M_{500}$ relation in the $i$ Sloan band. The empty squares in the figure indicate clusters with mass estimated from the dynamical analysis of the Sloan spectroscopic data. The filled points indicate systems with mass estimated from the $M-T_{\mathrm{X}}$ relation. The dot-dashed line is the best fit line obtained for the $\mathrm{O}$ sample. The dashed line is the best fit line for the $\mathrm{X}$ sample and the solid line is the result obtained from the E sample.

the sample with masses derived from the $M-T_{\mathrm{X}}$ relation (49 systems). Then we analyse the correlations obtained using all the clusters of the RASS-SDSS galaxy cluster sample with known mass (either the virial estimate from optical data, or, when this is not available, the mass derived from the X-ray temperature) for a total number of 102 systems (69 clusters with known optical mass +49 clusters with mass estimated from the temperature, with an overlap of 16 clusters). In the following tables, "O" (Optical sample) refers to the sample with optical masses, the "X" (X-ray sample) to the sample with mass derived from $T_{\mathrm{X}}$ and, finally, the "E" (Enlarged sample) refers to the sample of all the clusters with known mass.

Throughout the following analysis we consider only the relation obtained with the optical luminosity calculated in the $i$ Sloan band. All the results obtained in the other three SDSS optical bands $(g, r$ and $z$ ) are listed in the Appendix.

\subsection{Correlations of the optical and $X$-ray luminosities with the cluster mass}

Both the optical and the X-ray luminosity show a tight relation with the cluster mass (see Figs. 5 and 6, respectively for the $L_{\mathrm{op}}-M_{500}$ and $L_{\mathrm{X}}-M_{500}$ relations). The optical luminosity is estimated within the same radius as the mass, while $L_{\mathrm{X}}$ is the total X-ray luminosity of the system. The total $L_{\mathrm{X}}$ is not estimated within a fixed aperture but is calculated from the $\mathrm{X}$-ray luminosity radial profile. Table 1 lists the best fit values of the correlation for the different samples. In both the $L_{\mathrm{op}}-M$ and $L_{\mathrm{X}}-M$ relations the slope is flatter for the $\mathrm{O}$ sample than for the $\mathrm{X}$ sample. As a consequence, the best fit values of the

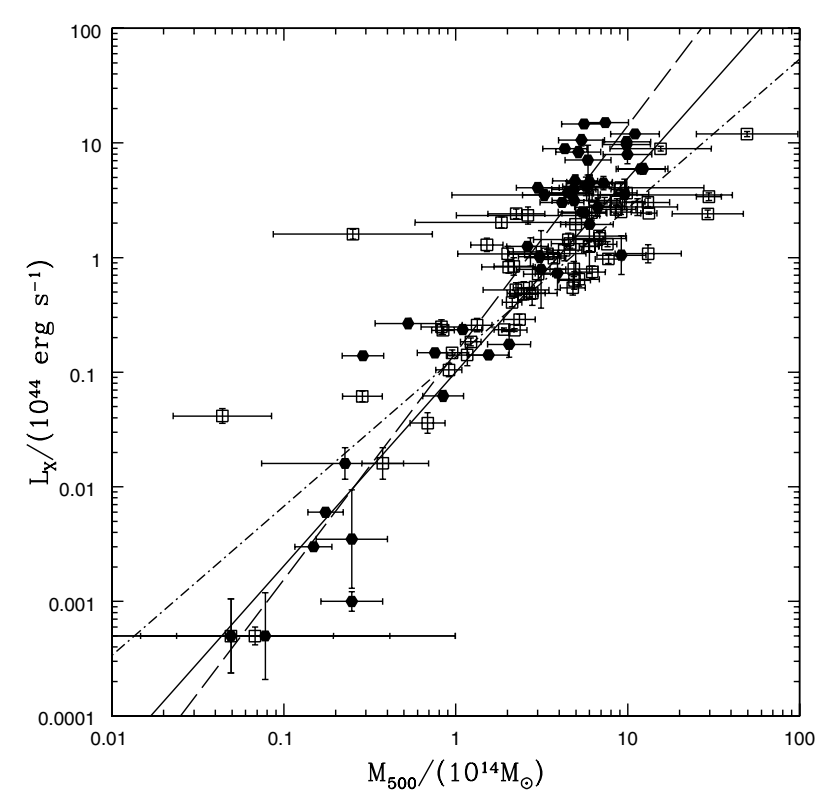

Fig. 6. $L_{\mathrm{X}}-M_{500}$ relation for the RASS-SDSS galaxy cluster sample. The empty squares in the figure are clusters with mass estimated from the dynamical analysis of the Sloan spectroscopic data. The filled points are systems with mass estimated from the $M-T_{\mathrm{X}}$ relation. The dot-dashed line is the best fit line obtained for the $\mathrm{O}$ sample. The dashed line is the best fit line for the X sample and the solid line is the result obtained from the E sample.

correlation obtained with the enlarged sample are the mean of the previous values. However, all the derived values of slope and normalization are in agreement within $1.5 \sigma$. The slope of $0.75 \pm 0.02$ of the $L_{\mathrm{op}}-M$ relation in the $B$ band studied by Girardi et al. (2002) is in very good agreement (within $1 \sigma$ ) with the slope of $0.81 \pm 0.04$ obtained with the $\mathrm{O}$ sample. The agreement is less good (within $3 \sigma$ ) for the slope obtained with the $\mathrm{X}$ and $\mathrm{E}$ samples. The values of slope and normalization of the $L_{\mathrm{X}}-M$ relation lie in the range of values given in $\mathrm{R} 02$. To recalibrate the $L_{\mathrm{X}}-M$ relation we add our enlarged sample to the sample of clusters of R02, obtaining a final sample of 198 clusters (106 from R02 + 102 from this work, with an overlap of 10) with known mass and X-ray luminosity. The best fit values of the correlation obtained with this sample are indicated by " $R+E$ " in Table 1 . The resulting slope of $1.5 \pm 0.05$ is in good agreement with the value obtained from the $\mathrm{E}$ sample. The correlation is shown in Fig. 7.

It is particularly interesting to compare the scatter of the $L_{\mathrm{op}}-M$ and $L_{\mathrm{X}}-M$ relations in order to understand which of the two observables, $L_{\mathrm{op}}$ and $L_{\mathrm{X}}$, shows the best correlation with the cluster mass. Table 1 lists three kinds of scatters evaluated for each correlation: the orthogonal scatter, which gives an estimate of the dispersion along the direction orthogonal to the best fit line, and the scatter in both variables. The orthogonal scatter in the $L_{\mathrm{op}}-M$ relation has a minimum value of $20 \%$ and a maximum of $30 \%$ in all the analysed correlations. The observable $L_{\mathrm{op}}$ can be used to predict the cluster mass within $r_{500}$ or $r_{200}$ with an accuracy in the range $40-50 \%$. The X-ray luminosity shows a less tight relation with the cluster mass for all the analysed correlations. In fact, the orthogonal scatter of the $L_{\mathrm{X}}-M$ relations lies in the range $38-50 \%$, while the mass can 
Table 1. The table lists the best fit parameters for the $L_{\mathrm{op}}-M, L_{\mathrm{X}}-M, L_{\mathrm{op}}-L_{\mathrm{X}}$ and $L_{\mathrm{op}}-r_{500 / 200}$ relations respectively for different samples of galaxy clusters and for different methods. O refers to the sample with masses estimated from the dynamical analysis performed with the optical spectroscopic data. X refers to the sample with masses estimated from the $M-T_{\mathrm{X}}$ relation. E refers to the enlarged sample, which comprises all the clusters in the RASS-SDSS galaxy cluster catalog with known mass. $R+E$ refers to the enlarged sample plus the sample of R02. The left side of the table lists the results obtained performing the dynamical analysis only for the red members od the system. The right side lists the best fit values of the correlations obtained using the results of the dynamical analysis applied to all the cluster members. The table lists three estimations of the scatter for each relation: $\sigma$ is the orthogonal scatter of the A-B relation, $\sigma_{A}$ is the scatter in the $A$ variable and $\sigma_{B}$ is the scatter in the $B$ variable. All the scatter values in the table are expressed in dex and all the errors are given at the $95 \%$ confidence level.

\begin{tabular}{|c|c|c|c|c|c|c|c|c|c|c|c|c|}
\hline \multicolumn{2}{|c|}{$A-B$ relation } & \multirow[t]{2}{*}{ Sample } & \multicolumn{5}{|c|}{ Red members } & \multicolumn{5}{|c|}{ All members } \\
\hline$A$ & $B$ & & $\alpha$ & $\beta$ & $\sigma$ & $\sigma_{B}$ & $\sigma_{A}$ & $\alpha$ & $\beta$ & $\sigma$ & $\sigma_{B}$ & $\sigma_{A}$ \\
\hline \multirow[t]{3}{*}{$M_{500}$} & $L_{\mathrm{op}}$ & $\mathrm{O}$ & $0.80 \pm 0.04$ & $-0.03 \pm 0.03$ & 0.12 & 0.16 & 0.16 & $0.81 \pm 0.04$ & $-0.09 \pm 0.03$ & 0.10 & 0.16 & 0.15 \\
\hline & & $\mathrm{X}$ & $0.96 \pm 0.05$ & $0.01 \pm 0.04$ & 0.09 & 0.15 & 0.16 & & & & & \\
\hline & & $\mathrm{E}$ & $0.91 \pm 0.04$ & $-0.03 \pm 0.03$ & 0.11 & 0.19 & 0.17 & $0.90 \pm 0.05$ & $-0.06 \pm 0.03$ & 0.11 & 0.17 & 0.16 \\
\hline \multirow[t]{3}{*}{$M_{200}$} & $L_{\mathrm{op}}$ & $\mathrm{O}$ & $0.79 \pm 0.04$ & $0.12 \pm 0.03$ & 0.13 & 0.16 & 0.15 & $0.79 \pm 0.04$ & $-0.05 \pm 0.04$ & 0.10 & 0.16 & 0.14 \\
\hline & & $\mathrm{X}$ & $1.05 \pm 0.07$ & $-0.04 \pm 0.06$ & 0.10 & 0.17 & 0.16 & & & & & \\
\hline & & $\mathrm{E}$ & $0.91 \pm 0.04$ & $0.05 \pm 0.04$ & 0.11 & 0.17 & 0.16 & $0.91 \pm 0.04$ & $-0.08 \pm 0.04$ & 0.12 & 0.19 & 0.17 \\
\hline \multirow[t]{4}{*}{$M_{500}$} & $L_{\mathrm{X}}$ & $\mathrm{O}$ & $1.30 \pm 0.09$ & $-0.77 \pm 0.09$ & 0.15 & 0.19 & 0.30 & $1.41 \pm 0.12$ & $-0.92 \pm 0.06$ & 0.18 & 0.19 & 0.32 \\
\hline & & $\mathrm{X}$ & $1.87 \pm 0.12$ & $-0.83 \pm 0.06$ & 0.14 & 0.19 & 0.29 & & & & & \\
\hline & & $\mathrm{E}$ & $1.68 \pm 0.09$ & $-0.88 \pm 0.06$ & 0.17 & 0.22 & 0.36 & $1.69 \pm 0.10$ & $-1.00 \pm 0.06$ & 0.19 & 0.23 & 0.43 \\
\hline & & $\mathrm{R}+\mathrm{E}$ & $1.50 \pm 0.05$ & $-0.38 \pm 0.03$ & 0.17 & 0.22 & 0.27 & & & & & \\
\hline \multirow[t]{4}{*}{$M_{200}$} & $L_{\mathrm{X}}$ & $\mathrm{O}$ & $1.30 \pm 0.09$ & $-0.95 \pm 0.07$ & 0.15 & 0.19 & 0.30 & $1.32 \pm 0.08$ & $-1.08 \pm 0.07$ & 0.17 & 0.18 & 0.30 \\
\hline & & $X$ & $1.98 \pm 0.13$ & $-1.24 \pm 0.10$ & 0.16 & 0.20 & 0.29 & & & & & \\
\hline & & $\mathrm{E}$ & $1.71 \pm 0.09$ & $-1.18 \pm 0.07$ & 0.17 & 0.21 & 0.35 & $1.66 \pm 0.09$ & $-1.27 \pm 0.07$ & 0.17 & 0.21 & 0.39 \\
\hline & & $\mathrm{R}+\mathrm{E}$ & $1.58 \pm 0.07$ & $-0.92 \pm 0.06$ & 0.16 & 0.26 & 0.30 & & & & & \\
\hline \multirow[t]{3}{*}{$L_{\mathrm{X}}$} & $L_{\mathrm{op}}$ & $\mathrm{O}$ & $0.60 \pm 0.05$ & $0.40 \pm 0.02$ & 0.12 & 0.30 & 0.16 & $0.63 \pm 0.04$ & $0.41 \pm 0.02$ & 0.14 & 0.29 & 0.17 \\
\hline & $\left(r_{500}\right)$ & $X$ & $0.53 \pm 0.03$ & $0.39 \pm 0.03$ & 0.16 & 0.33 & 0.19 & & & & & \\
\hline & & $\mathrm{E}$ & $0.54 \pm 0.03$ & $0.40 \pm 0.03$ & 0.14 & 0.33 & 0.18 & $0.55 \pm 0.03$ & $0.41 \pm 0.02$ & 0.15 & 0.33 & 0.18 \\
\hline \multirow[t]{3}{*}{$L_{\mathrm{X}}$} & $L_{\mathrm{op}}$ & $\mathrm{O}$ & $0.59 \pm 0.05$ & $0.55 \pm 0.02$ & 0.13 & 0.28 & 0.16 & $0.64 \pm 0.04$ & $0.57 \pm 0.02$ & 0.15 & 0.27 & 0.17 \\
\hline & $\left(r_{200}\right)$ & $\mathrm{X}$ & $0.55 \pm 0.04$ & $0.56 \pm 0.03$ & 0.19 & 0.35 & 0.22 & & & & & \\
\hline & & $\mathrm{E}$ & $0.56 \pm 0.03$ & $0.56 \pm 0.03$ & 0.16 & 0.32 & 0.18 & $0.58 \pm 0.03$ & $0.57 \pm 0.02$ & 0.17 & 0.31 & 0.19 \\
\hline \multirow[t]{3}{*}{$r_{500}$} & $L_{\mathrm{op}}$ & $\mathrm{O}$ & $2.28 \pm 0.14$ & $0.31 \pm 0.02$ & 0.05 & 0.05 & 0.15 & $2.26 \pm 0.13$ & $0.33 \pm 0.04$ & 0.05 & 0.06 & 0.14 \\
\hline & & $X$ & $2.95 \pm 0.15$ & $0.43 \pm 0.02$ & 0.04 & 0.05 & 0.14 & & & & & \\
\hline & & $\mathrm{E}$ & $2.53 \pm 0.12$ & $0.36 \pm 0.02$ & 0.05 & 0.07 & 0.17 & $2.50 \pm 0.13$ & $0.38 \pm 0.05$ & 0.05 & 0.06 & 0.17 \\
\hline \multirow[t]{3}{*}{$r_{200}$} & $L_{\mathrm{op}}$ & $\mathrm{O}$ & $2.25 \pm 0.13$ & $0.07 \pm 0.04$ & 0.06 & 0.07 & 0.14 & $2.28 \pm 0.14$ & $0.08 \pm 0.04$ & 0.06 & 0.08 & 0.14 \\
\hline & & $\mathrm{X}$ & $2.88 \pm 0.18$ & $0.06 \pm 0.05$ & 0.06 & 0.08 & 0.17 & & & & & \\
\hline & & $\mathrm{E}$ & $2.49 \pm 0.12$ & $0.06 \pm 0.04$ & 0.07 & 0.09 & 0.17 & $2.52 \pm 0.11$ & $0.07 \pm 0.04$ & 0.07 & 0.08 & 0.17 \\
\hline
\end{tabular}

be predicted from the X-ray luminosity with an accuracy in the range $55-65 \%$. These values are in agreement with the results of R02 and are confirmed by the correlation obtained with the " $\mathrm{R}+\mathrm{E}$ " sample, as indicated in Table 1. The dispersion along the $L_{X}$ axis is much larger (more than $90 \%$ ) and is due to the propagation of the errors, since $\sigma_{L_{\mathrm{X}}} \sim \alpha \times \sigma_{M}$. The difference in the scatter observed in the two relations cannot be explained by the measurement errors, since the average error in the $L_{\mathrm{op}}$ measure is around $15 \%$ and is comparable to the average measurement error in $L_{\mathrm{X}}$ ( around 10\%).

\subsection{The scatter in the $L_{\mathrm{op}}-M$ and $L_{\mathrm{x}}-M$ relations}

A possible explanation for the large scatter of the $L_{X}-M$ relation is the presence of a large number of groups in our samples. In fact measuring the mass and the X-ray luminosity for low-mass systems is not an easy task. However, the low-mass systems do not increase the scatter in the $L_{\mathrm{op}}-M$ relation, as they should if their masses were not measured correctly. Moreover, the scatter of the $L_{\mathrm{X}}-M$ relation is the same in all the mass ranges, since including or excluding the groups in our analysis does not change the amount of scatter in the correlations. A more plausible explanation for the larger scatter in the $L_{\mathrm{X}}-M$ relation in comparison to the $L_{\mathrm{op}}-M$ relation, is the presence of a large number of cool-core (once named "cooling flow") clusters in the analysed sample. In fact, the presence of a cool core in a cluster could cause an increase of the observed $\mathrm{X}$-ray luminosity for a given cluster mass. In principle even the mass estimated from the ICM temperature could be affected by this effect. In fact, Markevitch (1998, hereafter M98) has shown that the presence of a cool core in a system can significantly affect the temperature estimation if the cool core region 


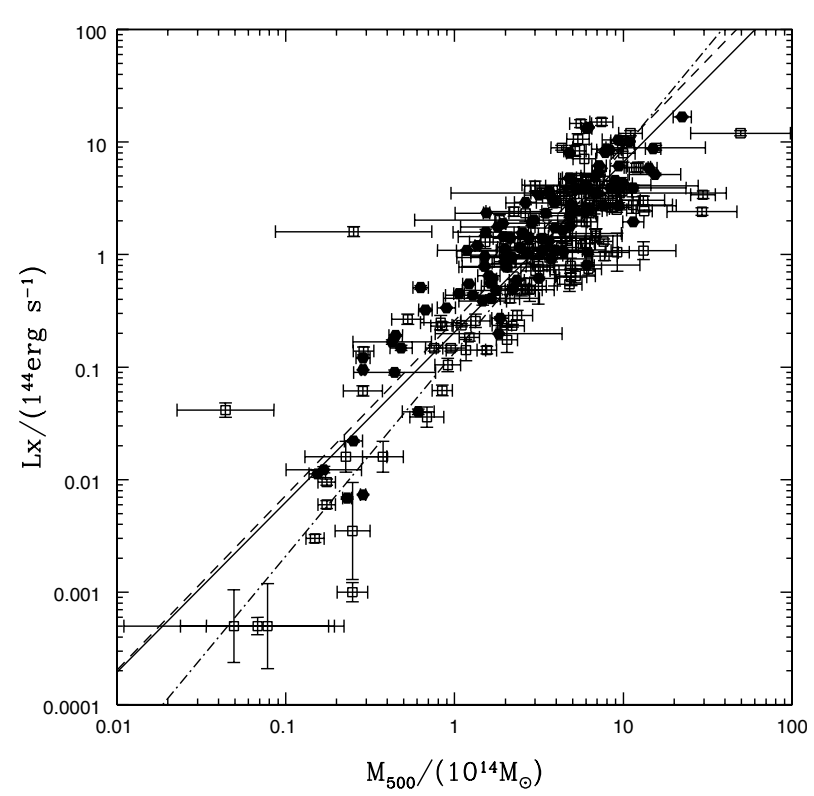

Fig. 7. $L_{\mathrm{X}}-M_{500}$ relation for the RASS-SDSS galaxy cluster sample plus the cluster sample of R02. The empty squares are the E clusters and the filled hexagons are the clusters of R02. The dot-dashed line is the best fit line obtained for the $\mathrm{E}$ sample. The dashed line is the best fit line for the R02 sample and the solid line is the result obtained from the "E+R" sample.

is not excised. However, the scatter observed in the $L_{\mathrm{X}}-M$ relation obtained with the $\mathrm{X}$ sample and with the R02 sample, in which the mass is calculated using the temperature, is exactly the same as the scatter observed in the $L_{X}-M$ relation obtained with the $\mathrm{O}$ sample, in which the mass estimation is not influenced by the presence of a cool core. Thus, we do not expect that the presence of a cool core affects the mass in our correlation. Therefore, in Fig. 7 the cool-core clusters should move to higher X-ray luminosity but not to higher mass. We call this effect the "cool core" effect throughout the paper.

Unfortunately our data are not able to fully explore this effect. To calculate the amount of scatter due to the cool-core effect on the X-ray luminosity we must use the cluster sample of M98. In this sample the X-ray luminosities and temperatures have been corrected for the cool core effect. We retrieve the masses of 33 of the 35 clusters of that sample from R02. The masses taken from R02 have all been calculated with the corrected temperature of M98. In Fig. 8 we show the $L_{\mathrm{X}}-M$ relation obtained using a cool-core-corrected X-ray luminosity. Panels $a$ and $b$ show the $L_{\mathrm{X}}-M$ relation given by the X-ray luminosity calculated in the $0.1-2.4 \mathrm{keV}$ energy band within $1.4 \mathrm{Mpc}$ from the cluster center with $M_{500}$ and $M_{200}$ respectively. Panels $c$ and $d$ show the same relation given by the total X-ray luminosity of the system in the same energy band and $M_{200}$. The total $L_{\mathrm{X}}$ is taken from R02 and is calculated within $r_{200}$ with a method similar to the method used to estimate the X-ray luminosity in the RASS-SDSS sample. Therefore, it should give a robust estimate of the total X-ray emission of the system. Panel $c$ shows the $L_{X}-M$ relation with uncorrected X-ray luminosity, while panel $d$ shows the $L_{\mathrm{X}}-M$ relation obtained using the X-ray luminosity corrected for the cool core effect. The correction is obtained by
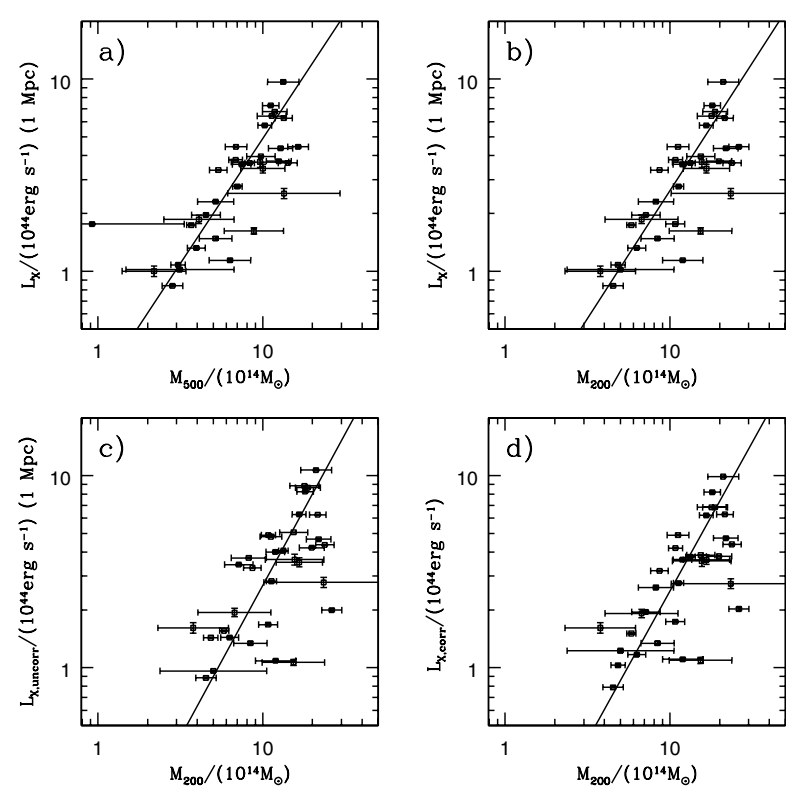

Fig. 8. $L_{X}-M_{500}$ relation for the sample of M98. The $L_{X}-M$ relation is obtained using a cool-core-corrected X-ray luminosity. Panels a) and b) show the $L_{\mathrm{X}}-M$ relation given by the X-ray luminosity calculated in the 0.1-2.4 keV energy band within $1.4 \mathrm{Mpc}$ from the cluster center with $M_{500}$ and $M_{200}$ respectively. The X-ray luminosity is taken from M98, while the mass is taken from R02. R02 used the coolcore-corrected temperature of M98 to calculate the mass. Panels c) and d) show the same relation given by the total X-ray luminosity of the system (calculated within the physical size of the system) in the $0.1-2.4 \mathrm{keV}$ energy band and $M_{200}$. The total $L_{\mathrm{X}}$ is taken from R02 and is calculated with the same method used to estimate the X-ray luminosity in the RASS-SDSS sample. Panel c) shows the $L_{\mathrm{X}}-M$ relation with uncorrected $X$-ray luminosity, while panel d) shows the $L_{\mathrm{X}}-M$ relation obtained using the X-ray luminosity corrected for the cool-core effect. The correction is obtained comparing the corrected and uncorrected $L_{\mathrm{X}}$ retrieved in M98.

comparing the corrected and uncorrected $L_{\mathrm{X}}$ calculated by M98. M98 removed the effect due to the presence of a cool core by the excision of the cool core region. They assumed that a $70 \mathrm{kpc}$ radius contains most of the cool core emission in clusters that do not have an extremely cool core, like those in the sample considered here. Therefore, to do the excision in a uniform manner, for all clusters, regions of $70 \mathrm{kpc}$ radius centered on the main brightness peak were masked, and the resulting fluxes and luminosities were multiplied by 1.06 to account for the flux inside the masked region, assuming an average $\beta$ model for the cluster X-ray brightness. Therefore, subtracting the corrected from the uncorrected X-ray luminosity gives the amount of cool-core correction applied by M98. We use the same amount to correct the total X-ray luminosities given by R02.

As shown in Table 2, applying the correction for cool-core effect does not change the scatter of the relation. In fact, in the relation obtained using the total X-ray luminosity, the scatter along the $M_{200}$ axis is 0.13 dex before the correction and 0.12 dex after that. This means that the cool core correction reduces the scatter by only $3 \%$. As a matter of fact, for most of the clusters in the M98 sample the correction is of the same order. Moreover, it is important to stress here that even the 
Table 2. The table lists the best fit values for the $L_{\mathrm{X}}-M$ relations. The first three lines list the relations obtained with the M98 data: $L_{\mathrm{X}}-M_{500}$ with $L_{\mathrm{X}}$ calculated in 0.1-2.4 keV energy band, within $1.4 \mathrm{Mpc}$ from the cluster center and corrected for cool-core effect, $L_{\mathrm{X}}-M_{200}\left(L_{\mathrm{X}}\right.$ before), $L_{\mathrm{X}}(\mathrm{Bol})-M_{200}$ with the bolometric X-ray luminosity calculated within $1.4 \mathrm{Mpc}$ from the cluster center and corrected for cool-core effect. The last two lines list the correlations obtained with the total X-ray luminosity taken from R02 corrected and uncorrected for the cool-core effect respectively. The table lists three estimations of the scatter for each relation: $\sigma$ is the orthogonal scatter of the $A-B$ relation, $\sigma_{A}$ is the scatter in the $A$ variable and $\sigma_{B}$ is the scatter in the $B$ variable. All the scatter values in the table are expressed in dex and all the errors are given at the $95 \%$ confidence level.

\begin{tabular}{c|ccccc}
\hline \hline & $\alpha$ & $\beta$ & $\sigma$ & $\sigma_{B}$ & $\sigma_{A}$ \\
\hline$L_{\mathrm{X}}(0.1-2.4 \mathrm{keV})-M_{500}(1.4 \mathrm{Mpc})$ & $1.30 \pm 0.12$ & $-0.61 \pm 0.10$ & 0.10 & 0.11 & 0.20 \\
$L_{\mathrm{X}}(0.1-2.4 \mathrm{keV})-M_{200}(1.4 \mathrm{Mpc})$ & $1.33 \pm 0.13$ & $-0.91 \pm 0.14$ & 0.10 & 0.11 & 0.14 \\
$L_{\mathrm{X}}(\mathrm{Bol})-M_{200}(1.4 \mathrm{Mpc})$ & $2.01 \pm 0.20$ & $-1.35 \pm 0.22$ & 0.10 & 0.11 & 0.20 \\
$L_{X, \text { corr }}(0.1-2.4 \mathrm{keV})-M_{200}($ tot $)$ & $1.55 \pm 0.19$ & $-1.15 \pm 0.20$ & 0.11 & 0.12 & 0.17 \\
$L_{X, \text { uncorr }}(0.1-2.4 \mathrm{keV})-M_{200}($ tot $)$ & $1.58 \pm 0.23$ & $-1.15 \pm 0.24$ & 0.11 & 0.13 & 0.18 \\
\hline
\end{tabular}

scatter of the $L_{\mathrm{X}}-M$ relation obtained in this analysis with the uncorrected X-ray luminosity is much lower than the dispersion obtained with the the RASS-SDSS galaxy cluster catalog, which is a sample more than 3 times as large. In fact, the sample of M98 covers a very small range in mass and Xray luminosity, only one order of magnitude in both variables. Hence, the statistical significance of the result is very low and it cannot be taken as a robust result, since it does not seem to represent the behavior of the $L_{\mathrm{X}}-M$ relation obtained with much larger samples of clusters. Therefore, it is not clear if the presence of a large number of cool core clusters in our sample and in the sample of R02 could really contribute to the scatter in the $L_{\mathrm{X}}-M$ relation. As a last point, we note that replacing the $L_{X}$ calculated in the ROSAT energy band $(0.1-2.4 \mathrm{keV})$ with the bolometric luminosity does not change at all the scatter in the relation. The bolometric X-ray luminosity is taken from M98. The slope of the $L_{\mathrm{X}}$, bol $-M_{200}$ relation is steeper than the $L_{\mathrm{X}}$, ROSAT $-M_{200}$ relation, as expected (the bolometric correction is smaller for the faint X-ray clusters than in the bright ones), while the orthogonal scatter and the dispersion along the $M_{200}$ axis are unchanged. The scatter in $L_{\mathrm{X}}$ changes because of the slope, since $\sigma_{B} \sim \alpha \times \sigma_{A}$. In conclusion, to really understand the nature of the scatter in the $L_{\mathrm{X}}-M$ relation and its connection to the cool core correction to the X-ray luminosity, the analysis should be done with a cluster sample much larger than the M98 sample and with a much more extended range in mass and $L_{\mathrm{X}}$.

Finally, let us consider the possibility that the better behavior of the optical luminosity as a mass predictor, in comparison with $L_{\mathrm{X}}$ could be due to the fact that $L_{\mathrm{op}}$ is calculated within the same aperture as the mass $\left(r_{500}\right.$ and $\left.r_{200}\right)$ while $L_{\mathrm{X}}$ is estimated within a variable aperture. R02 calculate the mass within $r_{200}$ and yet obtain the same scatter we observe in the $L_{\mathrm{X}}-M$ relation. Therefore, the scatter in the $L_{\mathrm{X}}-M$ relation does not seem to depend on the limiting radius used to compute $L_{\mathrm{X}}$. The observed dispersion in the $L_{\mathrm{X}}-M$ relation is most probably due to variations in the compactness of clusters. The dichotomy of compact $\mathrm{cD}$ clusters (often associated with the cooling flow signatures) and less compact non-cD clusters is more pronounced than just an excess of X-ray flux in the central $70 \mathrm{kpc}$ region (as used in the above correction for cooling flows). This is indicated for example by the work of Jones \& Forman (1984) and Ota \& Mitsuda (2002) and has been discussed by Fabian et al. (1994). Because of the strong quadratic dependence of the X-ray emission on the gas density this variation in compactness is observed in an amplified way in the $\mathrm{X}$-ray luminosity variation.

The most important and interesting conclusion of this analysis is that both the optical and the X-ray luminosity show extremely good relations with the cluster mass within $r_{500}$ and $r_{200}$. The $L_{\mathrm{op}}-M$ relation is tighter than the $L_{\mathrm{X}}-M$ relation and the optical luminosity can be used as mass estimator with an average accuracy of $40 \%$. The X-ray luminosity can predict the cluster mass with an accuracy of $55 \%$ on average. The presence of a large number of cool core clusters in our sample does not seem to be the cause of the larger scatter in the $L_{\mathrm{X}}-M$ relation than in the $L_{\mathrm{op}}-M$ relation.

Our result is in excellent agreement with that obtained by Lin et al. (2003), who used the $K$-band luminosity as a mass predictor, and found an average accuracy of $45 \%$.

On the basis of these results the scatter of the $L_{\mathrm{op}}-L_{\mathrm{X}}$ relation has a natural explanation. As shown in Table 1 the values of all the estimated scatter values are very close to the values calculated for the $L_{\mathrm{X}}-M$ relation. Therefore, we conclude that the scatter in the $L_{\mathrm{op}}-L_{\mathrm{X}}$ relation (Fig. 9) is mostly derived from the scatter in the $L_{\mathrm{X}}-M$ relation.

\subsection{The mass-to-light ratio}

To conclude the analysis of the relation between the optical luminosity and the cluster mass, we consider the mass-to-light ratio, $M / L$, as a function of the cluster mass.

Previous analyses have shown that, in general, $M / L$ increases with the cluster mass. Assuming a relation of the type $M / L \propto M^{\alpha}$, and adopting the usual scaling relations between mass and X-ray temperature or velocity dispersion, when needed, most authors have found $\alpha \simeq 0.25 \pm 0.1$, in both optical and near-infrared bands, and over a very large mass range, from loose groups to rich clusters of galaxies (Adami et al. 1998; Bahcall \& Comerford 2002; Girardi et al. 2002; Lin et al. 2003, 2004; Rines et al. 2004; Ramella et al. 2004; see however Kochanek et al. 2003, for a discordant result). 


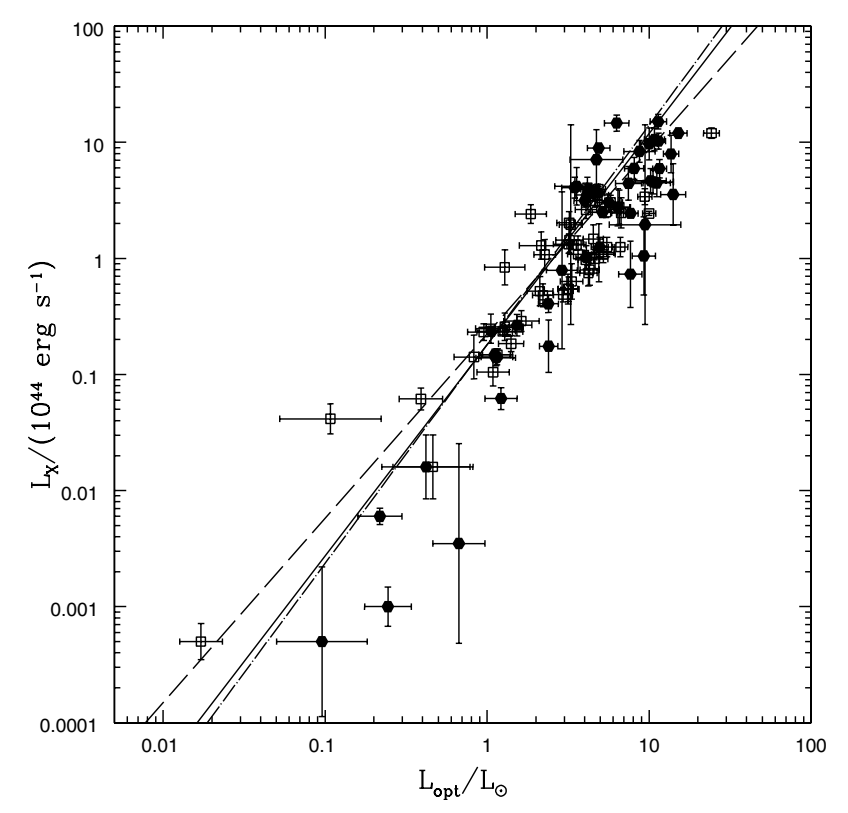

Fig. 9. $L_{\mathrm{op}}-L_{\mathrm{X}}$ relation. The optical luminosities are calculated within $r_{500}$. The empty squares in the figure are clusters with mass estimated from the dynamical analysis of the Sloan spectroscopic data. The filled points are systems with $r_{500}$ estimated from mass obtained with the $M-T_{\mathrm{X}}$ relation. The dot-dashed line is the best fit line obtained for the $\mathrm{O}$ sample. The dashed line is the best fit line for the $\mathrm{X}$ sample and the solid line is the result obtained from the E sample.

Our result is shown in Fig. 10, where we plot $M / L$ (in the $i$ band) calculated within $r_{500}$, versus $M_{500}$. An increase of the mass-to-light ratio with the mass is clearly visible. The existence of a correlation is confirmed by a Spearman correlation analysis (the correlation cofficient is 0.42 , corresponding to a probability of only $7 \times 10^{-6}$ that the two quantities are not correlated). In order to quantify the relation between mass and luminosity, we prefer to use the $L_{\mathrm{op}}-M$ relation directly. In fact, since $M / L$ is defined as a function of $M$ and $L$, it is not correct to search for the best-fitting relation of $M / L$ versus $M$ or $L$. The $L_{\mathrm{op}}-M$ relation implies $M / L \propto M^{0.2 \pm 0.08}$. Therefore the mass-to-light ratio of galaxy clusters is not constant, but (slightly) increases with the cluster mass. Our relation (derived in the $i$ band) is clearly consistent with the relations found in other bands ( $B$-band, Girardi et al. 2002; $V$-band, Bahcall \& Comerford 2002; $R$-band, Adami et al. 1998; $K$-band, Lin et al. 2003, 2004; Rines et al. 2004; Ramella et al. 2004), and, as a matter of fact, we checked that similar $M / L$ vs. $M$ dependencies are found in the other bands of the SDSS.

The fact that the $M / L$ vs. $M$ relation is wavelength independent clearly rules out the explanation provided by Bahcall \& Comerford (2002), namely that more massive clusters have a larger $M / L$ because their galaxies contain older stellar populations, on average, than galaxies that are members of less massive clusters. The most likely explanation for this $M / L$ variation with $M$ has been provided by Lin et al. (2003): the overall star formation efficiency must be a decreasing function of the cluster mass.

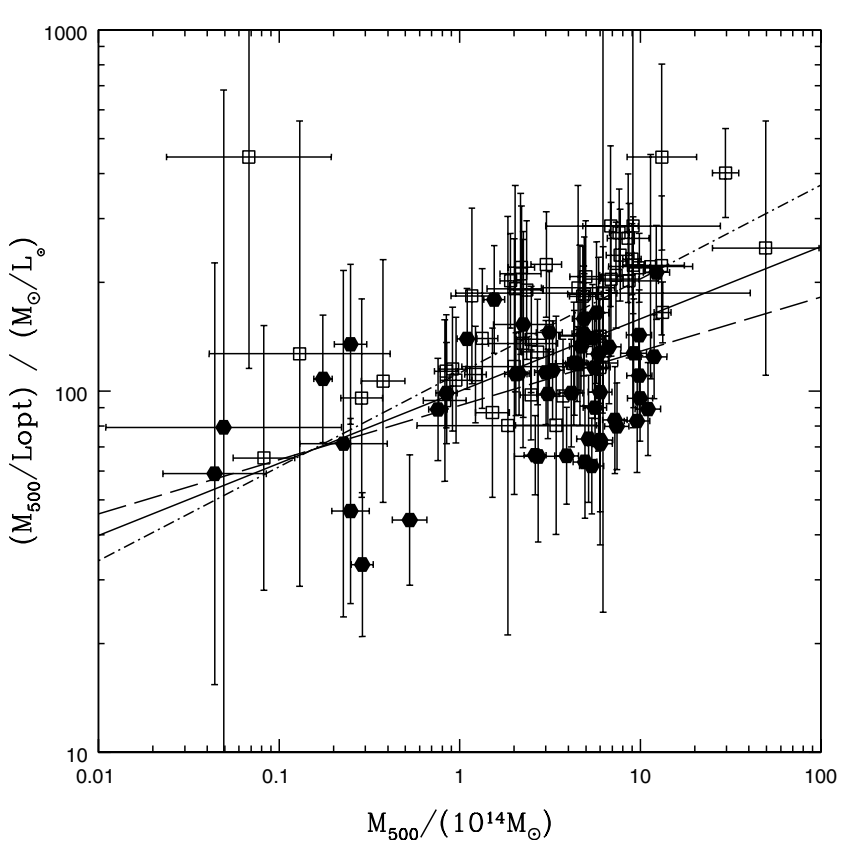

Fig. 10. $M / L-M$ relation. The mass-to-light ratio is calculated in the $i$ Sloan band. Masses and optical luminosities are calculated within $r_{500}$. The empty squares in the figure are clusters with masses estimated from the dynamical analysis of the Sloan spectroscopic data. The filled points are systems with masses estimated from the $M-T_{\mathrm{X}}$ relation. The dot-dashed line is the best fit line obtained for the $\mathrm{O}$ sample. The dashed line is the best fit line for the $\mathrm{X}$ sample and the solid line is the result obtained from the E sample.

\subsection{Correlations of the optical and the X-ray luminosities with the cluster temperature and velocity dispersion.}

The $\mathrm{X}$-ray temperature, $T_{\mathrm{X}}$, and the cluster velocity dispersion, $\sigma_{V}$, have both been used as key measures of cluster properties and in particular of the cluster mass. Given the excellent correlation of the optical luminosity with the mass, $L_{\mathrm{op}}$ should also correlate with, and have predictive power for, these two quantities. Table 3 summarizes the results obtained by correlating the optical luminosity in the $i$ Sloan band within $r_{500}$ and $r_{200}$ with $T_{\mathrm{X}}$ and $\sigma_{V}$. As shown by Figs. 11 and 12, the optical luminosity correlates very well with both $T_{\mathrm{X}}$ and $\sigma_{V}$ with an orthogonal scatter of $22 \%$ and $15 \%$ respectevely. Moreover, $L_{\mathrm{op}}$ can predict $T_{\mathrm{X}}$ with 23-28\% accuracy and $\sigma_{V}$ with $17-23 \%$ accuracy. Table 3 contains the best fit results also for the $L_{X}-T_{X}$ and $L_{\mathrm{X}}-\sigma_{V}$ relations. The best fit values of the relations are in perfect agreement with the results of Ortiz-Gil et al. (2004), who used a subsample of the REFLEX sample.

The X-ray luminosities defined in the REFLEX catalog are calculated with the same method as used for the RASS-SDSS cluster catalog. The orthogonal scatter of the $L_{\mathrm{X}}-\sigma_{V}$ relation $(17 \%)$ is also in good agreement with Ortiz-Gil et al. (2004), if we consider the relation obtained in that work using only clusters with accurate $\sigma_{V}$ estimation. As shown by Figs. 13 and 14 , the X-ray luminosity also shows a tight correlation with both quantities and the scatter of the best fit line (25-30\% accuracy in the $T_{\mathrm{X}}$ prediction and 20-23\% accuracy in the $\sigma_{V}$ prediction) is very close to the results obtained for the optical 
Table 3. The table lists the best fit values for several correlations: $L_{\mathrm{op}}-\sigma_{V}, L_{\mathrm{X}}-\sigma_{V}, L_{\mathrm{op}}-T_{\mathrm{X}}$ and $L_{\mathrm{X}}-T_{\mathrm{X}}$. The table shows the results obtained with the dynamical analysis performed for the red members of the systems ("red m." in the table) and for the complete cluster membership ("all $\mathrm{m}$ ". in the table). The table lists three estimations of the scatter for each relation: $\sigma$ is the orthogonal scatter of the $A-B$ relation, $\sigma_{A}$ is the scatter in the $A$ variable and $\sigma_{B}$ is the scatter in the $B$ variable. All the scatter values in the table are expressed in dex and all the errors are given at the $95 \%$ confidence level.

\begin{tabular}{ccccccc}
\hline \hline & & $\alpha$ & $\beta$ & $\sigma$ & $\sigma_{B}$ & $\sigma_{A}$ \\
\hline$L_{\mathrm{op}}-\sigma_{V}\left(r_{500}\right)$ & red m. & $2.26 \pm 0.13$ & $-6.04 \pm 0.38$ & 0.06 & 0.07 & 0.16 \\
\hline & all m. & $2.36 \pm 0.13$ & $-6.29 \pm 0.37$ & 0.06 & 0.07 & 0.17 \\
\hline$L_{\mathrm{op}}-\sigma_{V}\left(r_{200}\right)$ & red m. & $2.33 \pm 0.16$ & $-6.02 \pm 0.45$ & 0.06 & 0.08 & 0.17 \\
\hline & all m. & $2.33 \pm 0.15$ & $-6.05 \pm 0.42$ & 0.07 & 0.09 & 0.17 \\
\hline$L_{\mathrm{X}}-\sigma_{V}$ & red m. & $3.60 \pm 0.29$ & $-10.22 \pm 0.80$ & 0.07 & 0.09 & 0.38 \\
\hline & all m. & $3.68 \pm 0.25$ & $-10.53 \pm 0.80$ & 0.08 & 0.08 & 0.40 \\
\hline$L_{\mathrm{op}}-T_{\mathrm{X}}$ & $r_{500}$ & $1.68 \pm 0.08$ & $-0.50 \pm 0.06$ & 0.08 & 0.09 & 0.15 \\
\hline & $r_{200}$ & $1.66 \pm 0.09$ & $-0.41 \pm 0.07$ & 0.09 & 0.12 & 0.17 \\
\hline$L_{\mathrm{X}}-T_{\mathrm{X}}$ & & $3.06 \pm 0.10$ & $-1.77 \pm 0.07$ & 0.07 & 0.10 & 0.29 \\
\hline
\end{tabular}

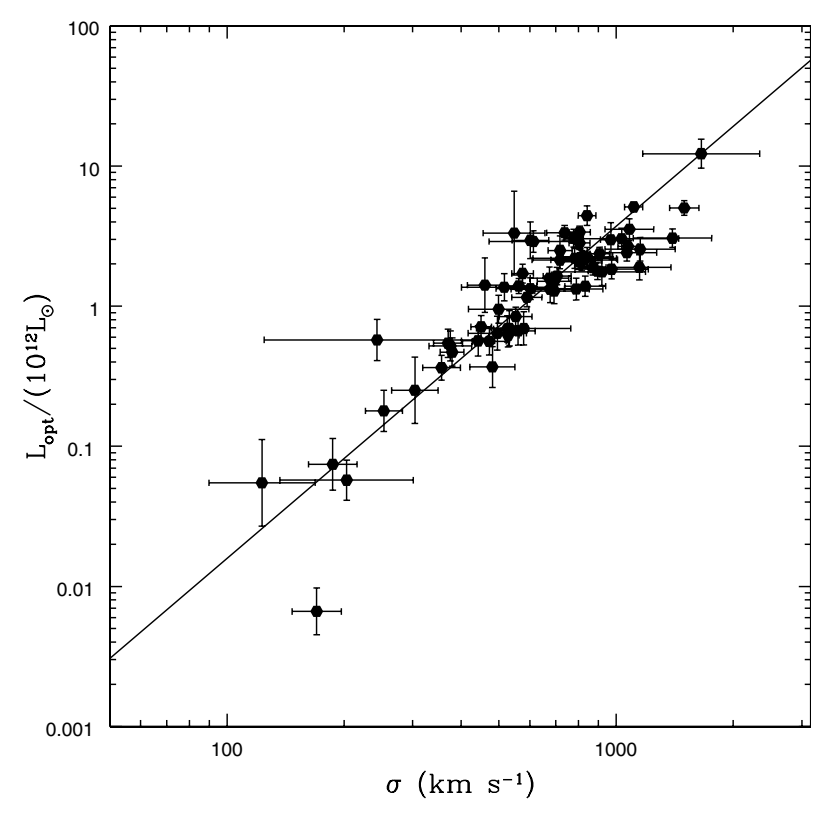

Fig. 11. $L_{\mathrm{op}}-\sigma_{V}$ relation. The optical luminosities are calculated within $r_{500}$. The best-fit line is also shown.

luminosity. $L_{\mathrm{op}}$ is a slightly better predictor than $L_{\mathrm{X}}$ with a $5 \%$ difference in the scatter. Note that, while $L_{\mathrm{op}}$ is a much better predictor of the cluster mass in comparison to $L_{\mathrm{X}}$, optical and $\mathrm{X}$-ray luminosities can predict approximately with the same accuracy $(20 \%)$ the intracluster temperature and the galaxy velocity dispersion. This different behavior of the scatter in the relations involving $L_{X}$ could be due to the dependence of the $\mathrm{X}$-ray luminosity and temperature on the cluster compactness. The cluster temperature is proportional to $M / R$, where $M$ is the cluster mass and $R$ is a characteristic radius of the system. Thus, $T_{\mathrm{X}}$ is related to mass with a weighting for compactness. As explained in the previous paragraph, $L_{X}$ is proportional to the gas density squared. This implies that, at given mass,

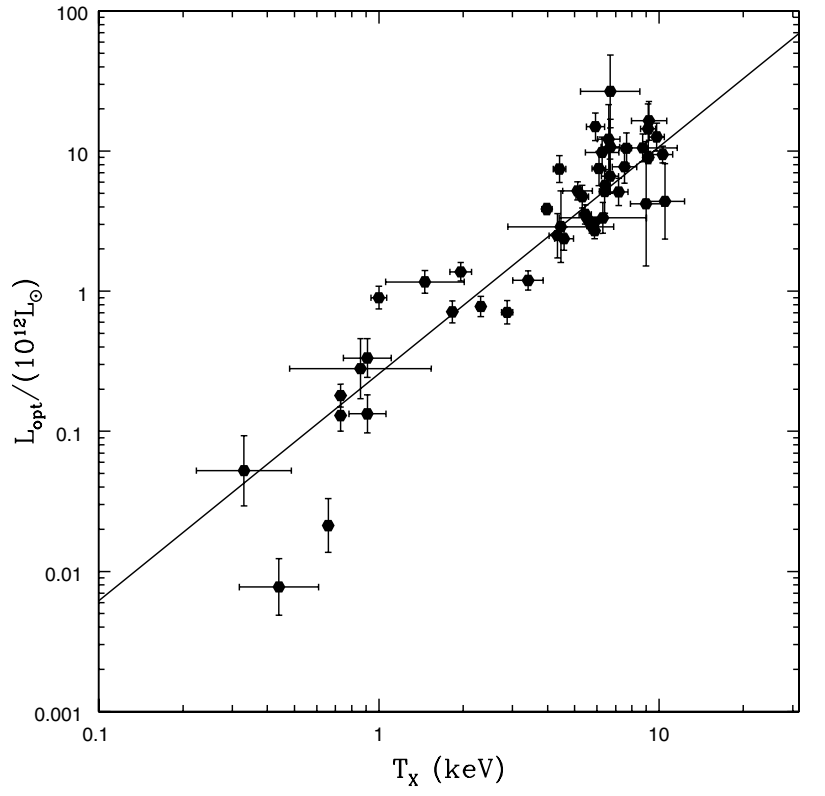

Fig. 12. $L_{\mathrm{op}}-T_{\mathrm{X}}$ relation. The optical luminosities are calculated within $r_{500}$. The best-fit line is also shown.

a compact cluster is much more X-ray bright than a less compact one. Therefore, the cluster compactness could enter the $L_{\mathrm{X}}-M$ relation as a third parameter, explaining the observed large scatter. However, since both $L_{\mathrm{X}}$ and $T_{\mathrm{X}}$ have a similar dependence on the compactness, the dispersion in the $L_{X}-T_{X}$ relation would not be affected. This would explain why $L_{\mathrm{op}}$ is a better estimator of the cluster mass than $L_{X}$, while optical and $\mathrm{X}$-ray luminosities have similar scatter in their relation with $T_{\mathrm{X}}$ and $\sigma_{V}$.

As in the case of the luminosity-mass relation, we investigate in more details the luminosity-temperature relation to understand which of the two luminosities is the best predictor of the other cluster parameters. We use the X-ray luminosity 


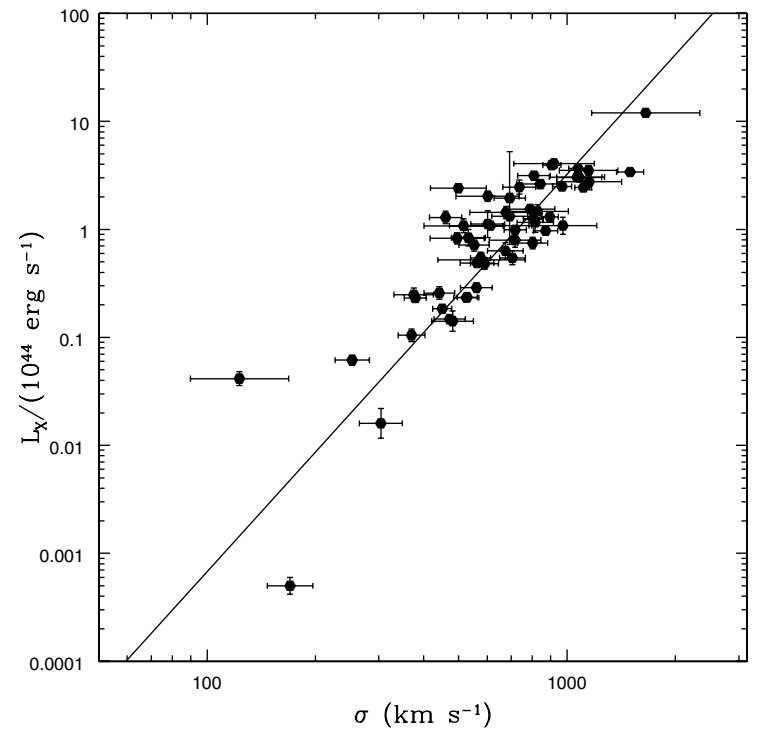

Fig. 13. $L_{\mathrm{X}}-\sigma_{V}$ relation. The best-fit line is also shown.

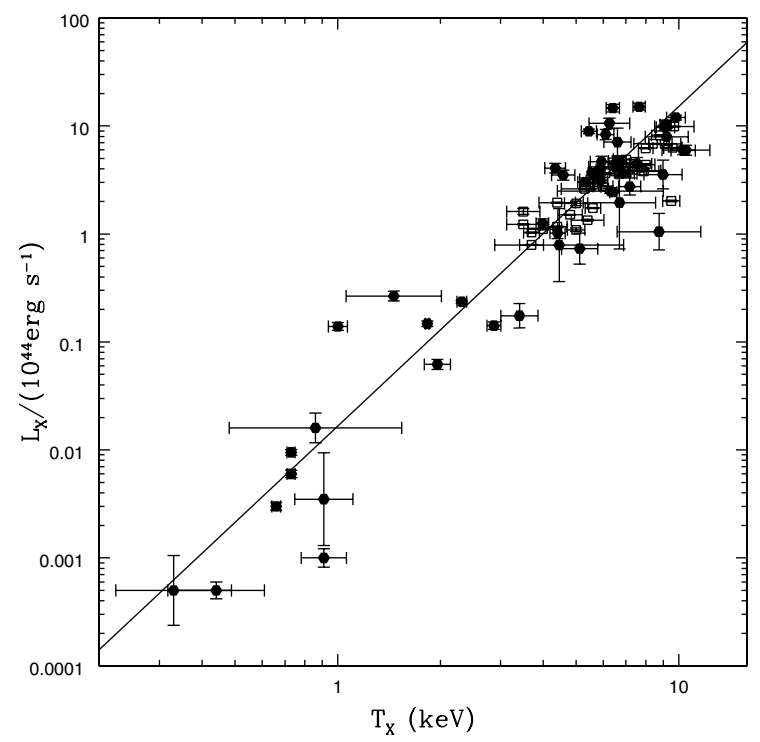

Fig. 14. $L_{X}-T_{X}$ relation. The filled points are the RASS-SDSS clusters with known ASCA temperature and the empty squares are the clusters of M98. The best-fit line is also shown.

(calculated within $1.4 \mathrm{Mpc}$ ) and the temperature from M98 to check the influence of the cool-core correction in the $L_{\mathrm{X}}-T_{\mathrm{X}}$ relation (Fig. 15). As shown in Table 4, using the cool-corecorrected X-ray luminosity and temperature lowers the scatter in the $T_{\mathrm{X}}$ variable by $6 \%$. We analyse the relation using also the total X-ray luminosity of R02 with and without the cool-core correction (panel $c$ and $d$, respectively, in Fig. 15), as we did for the $L_{\mathrm{X}}-M$ relation. In both cases, using the total $L_{\mathrm{X}}$ not only affects the slope of the relation, but also increases the scatter by $6 \%$ in comparison to the relations obtained using $L_{X}$ values from M98.

The slope of the $L_{\mathrm{X}}-T_{\mathrm{X}}$ relation obtained applying the cool-core-correction to the total luminosity and temperature is in perfect agreement with the results obtained previously with the subsample of "uncorrected" RASS-SDSS clusters, while the scatter is lower by $5 \%$. Such a reduction of the
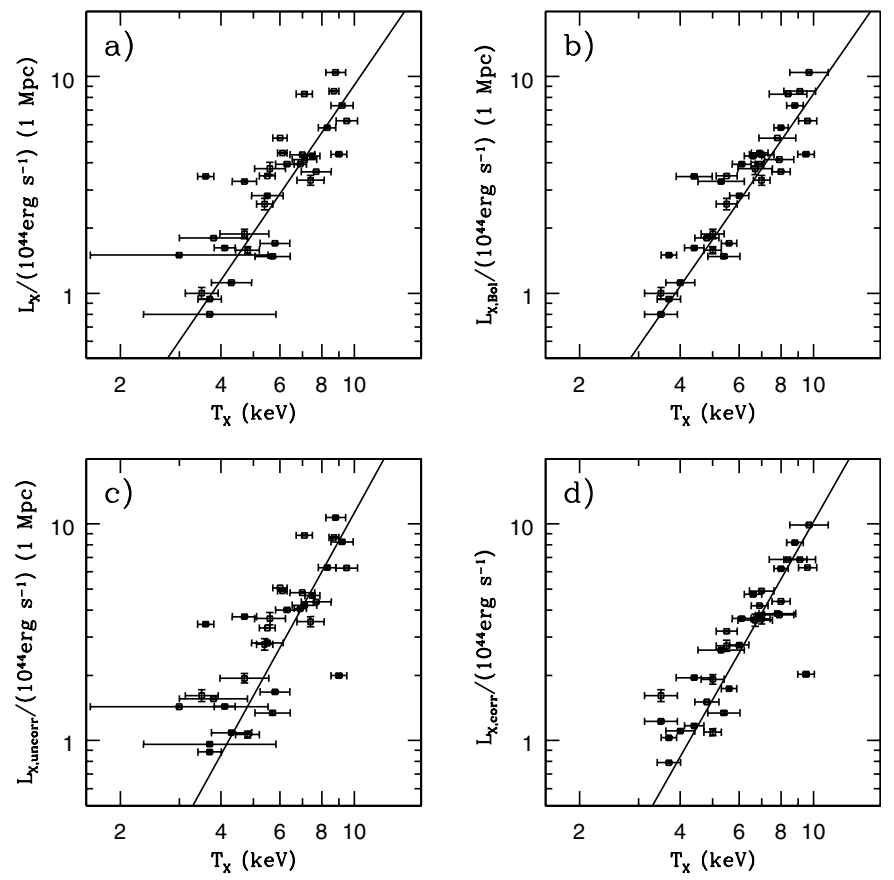

Fig. 15. The same as in Fig. 8 but for the $L_{\mathrm{X}}-T_{\mathrm{X}}$ relation.

scatter makes the X-ray luminosity a predictor of the X-ray temperature that is at least as good as the optical luminosity. However, a similar scatter reduction could in principle be expected for the $L_{\mathrm{op}}-T_{\mathrm{X}}$ relation also, since the cool-core correction affects X-ray temperatures more strongly than X-ray luminosities (M98). Unfortunately only few of the clusters of the M98 sample are in the sky region covered by the SDSS. Thus, we cannot check this possibility directly.

\section{Summary and conclusions}

We used the RASS-SDSS galaxy cluster sample to compare the quality of the optical and X-ray luminosity as predictors of other cluster properties such as the mass within $r_{500}$ and $r_{200}$, the velocity dispersion and the ICM temperature. The optical luminosity turns out to be a better predictor of the cluster mass than the X-ray luminosity. The knowledge of $L_{\mathrm{op}}$ make it possible to estimate the cluster mass with an average accuracy of $40 \%$, while $L_{X}$ can be used to predict the mass with an average accuracy of $55 \%$. We investigated the nature of the scatter of the $L_{\mathrm{X}}-M$ relation using a sample of clusters with X-ray luminosity corrected for the effect of a cool core at the center of the system. We concluded that this kind of effect can affect the scatter of the relation by at most $3 \%$ and, thus, it cannot explain the dispersion in the observed $L_{\mathrm{X}}-M$ relation, which is probably related to the variation in the compactness of the galaxy clusters. We conclude that a cluster optical luminosity is a better estimator of its mass than its X-ray luminosity. The optical luminosity is clearly a very useful and rather cheap estimator (in terms of observational resources required) given that it can be determined from ground-based photometric data only.

We also analysed the relations of the optical and X-ray luminosities with cluster velocity dispersions and X-ray temperatures. We found that both luminosities are strongly correlated 
Table 4. The table lists the best fit values for the $L_{\mathrm{X}}-T_{\mathrm{X}}$ relations. The first two lines list the relations obtained with the M98 data: $L_{\mathrm{X}}-T_{\mathrm{X}}$ with $L_{\mathrm{X}}$ calculated in the $0.1-2.4 \mathrm{keV}$ energy band, within $1 \mathrm{Mpc}$ from the cluster center and the temperature uncorrected for cool core effect, $L_{\mathrm{X}}-T_{\mathrm{X}}$ with the X-ray luminosity and temperature corrected for cool core effect. The last two lines list the correlations obtained with the total X-ray luminosity taken from R02 and the temperature of M98 corrected and uncorrected for cool core effect respectively. All the scatter values in the table are expressed in dex and have the same meaning as in the previous table.

\begin{tabular}{cccccc}
\hline \hline & $\alpha$ & $\beta$ & $\sigma$ & $\sigma_{A}$ & $\sigma_{B}$ \\
\hline$L_{\mathrm{X}}(0.1-2.4 \mathrm{keV})-T_{\mathrm{X}}(1.4 \mathrm{Mpc}$, uncorr $)$ & $2.59 \pm 0.38$ & $-1.48 \pm 0.31$ & 0.06 & 0.07 & 0.18 \\
$L_{\mathrm{X}}(0.1-2.4 \mathrm{keV})-T_{\mathrm{X}}(1.4 \mathrm{Mpc}$, corr $)$ & $2.26 \pm 0.19$ & $-1.30 \pm 0.15$ & 0.04 & 0.05 & 0.11 \\
$L_{\mathrm{X}, \text { corr }}(0.1-2.4 \mathrm{keV})-T_{\mathrm{X}}$ (tot, uncorr) & $3.30 \pm 0.67$ & $-2.06 \pm 0.54$ & 0.06 & 0.09 & 0.24 \\
$L_{\mathrm{X}, \text { uncorr }}(0.1-2.4 \mathrm{keV})-T_{\mathrm{X}}$ (tot, corr) & $2.80 \pm 0.38$ & $-1.75 \pm 0.30$ & 0.05 & 0.07 & 0.15 \\
\hline
\end{tabular}

with these cluster properties, and can be used to predict them with an average accuracy of $20 \%$. Using a sample of clusters with $L_{\mathrm{X}}$ and $T_{\mathrm{X}}$ corrected for the cool core effect, we find that the scatter in the $L_{\mathrm{X}}-T_{\mathrm{X}}$ relation is decreased by $5 \%$. Such a decrease is almost exclusively due to the correction applied to the X-ray temperature, since the cool-core correction has a negligible effect on the X-ray luminosity. Therefore, we expect a similar decrease of the scatter of the $L_{\mathrm{op}}-T_{\mathrm{X}}$ relation, when the $\mathrm{X}$-ray temperatures are corrected for the same cool-core effect. Unfortunately we cannot verify this expectation from our sample, since we lack the information to apply the cool-core correction to the clusters with known $L_{\mathrm{op}}$. We conclude that $L_{\mathrm{op}}$ and $L_{\mathrm{X}}$ can be used to predict the ICM temperature and the cluster velocity dispersion, at a similar level of accuracy.

The most important conclusion from our analysis is that the optical luminosity is a key measure of the fundamental properties of a galaxy cluster, such as its mass, velocity dispersion, and temperature. In this respect, the optical luminosity performs even better than the X-ray luminosity, which suggests that the mass distribution of a cluster is better traced by cluster galaxies than by intracluster gas (see, e.g., the discussion in Biviano \& Girardi 2003). The poorer performance of $L_{X}$ as a cluster mass predictor, relative to $L_{\mathrm{op}}$, is probably related to the variation in the compactness of the galaxy clusters.

Our conclusion is clearly in agreement with Lin et al.'s (2003) result, namely that the $K$-band luminosity is a good estimator of the cluster mass. On the other hand, our conclusion is at odds with the generally accepted view that the main physical properties of a cluster are more easily revealed in the X-ray than in the optical (e.g. Donahue et al. 2002). Such a view was established at an epoch when the lack of optical wide field surveys precluded a reliable determination of the optical luminosities of a large sample of clusters. With the advent of the Sloan Digital Sky survey, this problem is now overcome, and $L_{\mathrm{op}}$ can now be used to infer the fundamental physical properties of the many clusters being discovered within large optical surveys with improved cluster finding techniques (such as, e.g., the Red-Sequence Cluster Survey, Barrientos et al. 2003). In this context $L_{\mathrm{op}}$ becomes a very powerful means to do cosmological studies with galaxy clusters without the need of optical or X-ray spectroscopy.

Finally, we showed that the relation between mass and luminosity implies an increasing mass-to-light ratio, $M / L$, with increasing cluster mass. The dependence we found is in excellent agreement with previous results (Adami et al. 1998; Bahcall \& Comerford 2002; Girardi et al. 2002;
Lin et al. 2003, 2004; Rines et al. 2004; Ramella et al. 2004), and confirms the achromaticity of the effect. Hence, the effect cannot be explained by the different ages of the stellar populations of the galaxies in clusters of different masses (Bahcall \& Comerford 2002), but, rather, seems to indicate that the star formation efficiency decreases as the cluster mass increases (Lin et al. 2003).

The results obtained in this paper are applicable to nearby clusters since the RASS-SDSS galaxy cluster catalog comprises only X-ray clusters at $z \leq 0.3$. It would be very interesting to conduct the same analysis for a sample of high redshift clusters to study the evolution of the analysed relations and to compare again the quality of $L_{\mathrm{op}}$ and $L_{\mathrm{X}}$ as predictors of the other cluster properties.

The analysis conducted in this paper is based on a sample of clusters all detected in the X-ray, $90 \%$ of which are taken from $\mathrm{X}$-ray-selected galaxy cluster catalogs. It seems that optically bright clusters exist which are faint in the X-ray (Donahue et al. 2002). Hence, the selection criteria of the RASS-SDSS galaxy cluster catalog could in principle affect our results (see, e.g., Gilbank et al. 2004). To check how the selection criteria affect the correlations studied in this paper, we plan to repeat the same analyses for a sample of optically-selected clusters.

Acknowledgements. Funding for the creation and distribution of the SDSS Archive has been provided by the Alfred P. Sloan Foundation, the Participating Institutions, the National Aeronautics and Space Administration, the National Science Foundation, the US Department of Energy, the Japanese Monbukagakusho, and the Max Planck Society. The SDSS web site is http://www.sdss.org/. The SDSS is managed by the Astrophysical Research Consortium (ARC) for the Participating Institutions. The Participating Institutions are The University of Chicago, Fermilab, the Institute for Advanced Study, the Japan Participation Group, The Johns Hopkins University, Los Alamos National Laboratory, the Max-Planck-Institute for Astronomy (MPIA), the Max-Planck-Institute for Astrophysics (MPA), New Mexico State University, University of Pittsburgh, Princeton University, the United States Naval Observatory, and the University of Washington.

\section{Appendix A: Correlation of $L_{\text {op }}$ in the Sloan $g, r$ and $z$ bands with the cluster parameters}

We list in the table all the results obtained using the optical luminosity calculated in the $g, r$ and $z$ SDSS Sloan bands. The structure of the three tables in this appendix is similar to the tables in the text of the paper. For each analysed correlation we 


\begin{tabular}{|c|c|c|c|c|c|c|c|c|c|c|c|c|c|c|c|}
\hline \multicolumn{6}{|c|}{$g$ band } & \multicolumn{5}{|c|}{$r$ band } & \multicolumn{5}{|c|}{$z$ band } \\
\hline \multicolumn{16}{|c|}{$L_{\mathrm{op}}-M_{500}$} \\
\hline & $\alpha$ & $\beta$ & $\sigma$ & $\sigma_{M_{500}}$ & $\sigma_{L_{\mathrm{op}}}$ & $\alpha$ & $\beta$ & $\sigma$ & $\sigma_{M_{500}}$ & $\sigma_{L_{\mathrm{op}}}$ & $\alpha$ & $\beta$ & $\sigma$ & $\sigma_{M_{500}}$ & $\sigma_{L_{\mathrm{op}}}$ \\
\hline $\mathrm{O}$ & $0.81 \pm 0.05$ & $-0.30 \pm 0.03$ & 0.12 & 0.18 & 0.16 & $0.80 \pm 0.04$ & $-0.22 \pm 0.03$ & 0.11 & 0.16 & 0.16 & $0.91 \pm 0.04$ & $0.00 \pm 0.03$ & 0.13 & 0.18 & 0.19 \\
\hline $\mathrm{X}$ & $1.06 \pm 0.06$ & $-0.26 \pm 0.04$ & 0.14 & 0.22 & 0.17 & $1.08 \pm 0.06$ & $-0.18 \pm 0.04$ & 0.12 & 0.18 & 0.16 & $1.04 \pm 0.05$ & $0.07 \pm 0.04$ & 0.10 & 0.16 & 0.14 \\
\hline $\mathrm{E}$ & $0.90 \pm 0.04$ & $-0.31 \pm 0.03$ & 0.15 & 0.21 & 0.20 & $0.92 \pm 0.04$ & $-0.24 \pm 0.03$ & 0.13 & 0.20 & 0.20 & $0.79 \pm 0.04$ & $0.01 \pm 0.03$ & 0.10 & 0.16 & 0.15 \\
\hline \multicolumn{16}{|c|}{$L_{\mathrm{op}}-M_{200}$} \\
\hline & $\alpha$ & $\beta$ & $\sigma$ & $\sigma_{M_{200}}$ & $\sigma_{L_{\mathrm{op}}}$ & $\alpha$ & $\beta$ & $\sigma$ & $\sigma_{M_{200}}$ & $\sigma_{L_{\mathrm{op}}}$ & $\alpha$ & $\beta$ & $\sigma$ & $\sigma_{M_{200}}$ & $\sigma_{L_{\mathrm{op}}}$ \\
\hline $\mathrm{O}$ & $0.81 \pm 0.04$ & $-0.27 \pm 0.04$ & 0.13 & 0.18 & 0.15 & $0.80 \pm 0.04$ & $-0.18 \pm 0.04$ & 0.12 & 0.17 & 0.14 & $0.79 \pm 0.04$ & $0.06 \pm 0.04$ & 0.12 & 0.16 & 0.14 \\
\hline $\mathrm{X}$ & $1.08 \pm 0.08$ & $-0.25 \pm 0.06$ & 0.14 & 0.23 & 0.20 & $1.05 \pm 0.07$ & $-0.17 \pm 0.06$ & 0.12 & 0.20 & 0.17 & $1.02 \pm 0.06$ & $0.08 \pm 0.06$ & 0.10 & 0.18 & 0.16 \\
\hline $\mathrm{E}$ & $0.94 \pm 0.05$ & $-0.31 \pm 0.04$ & 0.16 & 0.23 & 0.20 & $0.94 \pm 0.04$ & $-0.24 \pm 0.04$ & 0.15 & 0.21 & 0.19 & $0.93 \pm 0.04$ & $0.00 \pm 0.04$ & 0.14 & 0.21 & 0.19 \\
\hline \multicolumn{16}{|c|}{$L_{\mathrm{op}}-L_{\mathrm{X}}\left(r_{500}\right)$} \\
\hline & $\alpha$ & $\beta$ & $\sigma$ & $\sigma_{L_{X}}$ & $\sigma_{L_{\mathrm{op}}}$ & $\alpha$ & $\beta$ & $\sigma$ & $\sigma_{L_{X}}$ & $\sigma_{L_{\mathrm{op}}}$ & $\alpha$ & $\beta$ & $\sigma$ & $\sigma_{L_{X}}$ & $\sigma_{L_{\mathrm{op}}}$ \\
\hline $\mathrm{O}$ & $0.62 \pm 0.05$ & $0.21 \pm 0.02$ & 0.15 & 0.31 & 0.18 & $0.63 \pm 0.04$ & $0.28 \pm 0.02$ & 0.14 & 0.29 & 0.17 & $0.63 \pm 0.04$ & $0.52 \pm 0.02$ & 0.13 & 0.28 & 0.16 \\
\hline $\mathrm{X}$ & $0.51 \pm 0.03$ & $0.19 \pm 0.03$ & 0.19 & 0.38 & 0.20 & $0.53 \pm 0.03$ & $0.27 \pm 0.03$ & 0.18 & 0.33 & 0.20 & $0.52 \pm 0.03$ & $0.49 \pm 0.03$ & 0.15 & 0.32 & 0.18 \\
\hline $\mathrm{E}$ & $0.55 \pm 0.03$ & $0.21 \pm 0.02$ & 0.17 & 0.35 & 0.19 & $0.56 \pm 0.03$ & $0.28 \pm 0.02$ & 0.15 & 0.32 & 0.18 & $0.56 \pm 0.03$ & $0.51 \pm 0.02$ & 0.14 & 0.31 & 0.17 \\
\hline \multicolumn{16}{|c|}{$L_{\mathrm{op}}-L_{\mathrm{X}}\left(r_{200}\right)$} \\
\hline & $\alpha$ & $\beta$ & $\sigma$ & $\sigma_{L_{X}}$ & $\sigma_{L_{\mathrm{op}}}$ & $\alpha$ & $\beta$ & $\sigma$ & $\sigma_{L_{X}}$ & $\sigma_{L_{\mathrm{op}}}$ & $\alpha$ & $\beta$ & $\bar{\sigma}$ & $\sigma_{L_{X}}$ & $\sigma_{L_{\mathrm{op}}}$ \\
\hline $\mathrm{O}$ & $0.63 \pm 0.05$ & $0.37 \pm 0.02$ & 0.17 & 0.28 & 0.18 & $0.64 \pm 0.04$ & $0.45 \pm 0.02$ & 0.15 & 0.26 & 0.17 & $0.64 \pm 0.04$ & $0.68 \pm 0.02$ & 0.15 & 0.25 & 0.16 \\
\hline $\mathrm{X}$ & $0.54 \pm 0.04$ & $0.37 \pm 0.03$ & 0.22 & 0.39 & 0.23 & $0.54 \pm 0.04$ & $0.44 \pm 0.03$ & 0.20 & 0.36 & 0.22 & $0.54 \pm 0.04$ & $0.67 \pm 0.03$ & 0.19 & 0.35 & 0.21 \\
\hline $\mathrm{E}$ & $0.57 \pm 0.03$ & $0.37 \pm 0.02$ & 0.19 & 0.34 & 0.19 & $0.58 \pm 0.03$ & $0.45 \pm 0.02$ & 0.17 & 0.31 & 0.19 & $0.58 \pm 0.03$ & $0.68 \pm 0.02$ & 0.16 & 0.30 & 0.18 \\
\hline \multicolumn{16}{|c|}{$L_{\mathrm{op}}-r_{500}$} \\
\hline & $\alpha$ & $\beta$ & $\sigma$ & $\sigma_{r_{500}}$ & $\sigma_{L_{\mathrm{op}}}$ & $\alpha$ & $\beta$ & $\sigma$ & $\sigma_{r_{500}}$ & $\sigma_{L_{\mathrm{op}}}$ & $\alpha$ & $\beta$ & $\sigma$ & $\sigma_{r_{500}}$ & $\sigma_{L_{\mathrm{op}}}$ \\
\hline $\mathrm{O}$ & $2.24 \pm 0.15$ & $0.11 \pm 0.02$ & 0.06 & 0.06 & 0.16 & $2.24 \pm 0.14$ & $0.19 \pm 0.02$ & 0.05 & 0.06 & 0.15 & $2.27 \pm 0.14$ & $0.42 \pm 0.02$ & 0.05 & 0.05 & 0.15 \\
\hline $\mathrm{X}$ & $2.95 \pm 0.16$ & $0.23 \pm 0.03$ & 0.06 & 0.07 & 0.17 & $2.97 \pm 0.16$ & $0.31 \pm 0.02$ & 0.05 & 0.06 & 0.15 & $2.93 \pm 0.15$ & $0.54 \pm 0.02$ & 0.04 & 0.05 & 0.14 \\
\hline $\mathrm{E}$ & $2.44 \pm 0.12$ & $0.14 \pm 0.02$ & 0.07 & 0.09 & 0.18 & $2.50 \pm 0.12$ & $0.23 \pm 0.02$ & 0.06 & 0.08 & 0.18 & $2.50 \pm 0.12$ & $0.47 \pm 0.02$ & 0.06 & 0.07 & 0.17 \\
\hline \multicolumn{16}{|c|}{$L_{\mathrm{op}}-r_{200}$} \\
\hline & $\alpha$ & $\beta$ & $\sigma$ & $\sigma_{r_{200}}$ & $\sigma_{L_{\mathrm{op}}}$ & $\alpha$ & $\beta$ & $\sigma$ & $\sigma_{r_{200}}$ & $\sigma_{L_{\mathrm{op}}}$ & $\alpha$ & $\beta$ & $\sigma$ & $\sigma_{r_{200}}$ & $\sigma_{L_{\mathrm{op}}}$ \\
\hline $\mathrm{O}$ & $2.27 \pm 0.15$ & $-0.14 \pm 0.04$ & 0.07 & 0.07 & 0.15 & $2.24 \pm 0.13$ & $-0.06 \pm 0.04$ & 0.06 & 0.07 & 0.14 & $2.26 \pm 0.13$ & $0.17 \pm 0.04$ & 0.06 & 0.06 & 0.14 \\
\hline $\mathrm{X}$ & $2.27 \pm 0.15$ & $-0.14 \pm 0.04$ & 0.07 & 0.07 & 0.15 & $2.85 \pm 0.18$ & $-0.05 \pm 0.05$ & 0.06 & 0.09 & 0.17 & $2.81 \pm 0.18$ & $0.19 \pm 0.05$ & 0.06 & 0.08 & 0.16 \\
\hline $\mathrm{E}$ & $2.44 \pm 0.13$ & $-0.14 \pm 0.04$ & 0.08 & 0.11 & 0.18 & $2.47 \pm 0.12$ & $-0.06 \pm 0.04$ & 0.07 & 0.09 & 0.17 & $2.45 \pm 0.12$ & $0.18 \pm 0.04$ & 0.07 & 0.09 & 0.17 \\
\hline \multicolumn{16}{|c|}{$L_{\mathrm{op}}-\sigma_{V}$} \\
\hline & $\alpha$ & $\beta$ & $\sigma$ & $\sigma_{\sigma_{V}}$ & $\sigma_{L_{\mathrm{op}}}$ & $\alpha$ & $\beta$ & $\sigma$ & $\sigma_{\sigma_{V}}$ & $\sigma_{L_{\mathrm{op}}}$ & $\alpha$ & $\beta$ & $\sigma$ & $\sigma_{\sigma_{V}}$ & $\sigma_{L_{\mathrm{op}}}$ \\
\hline$r_{500}$ & $2.37 \pm 0.15$ & $-6.54 \pm 0.41$ & 0.06 & 0.07 & 0.18 & $2.35 \pm 0.14$ & $-6.39 \pm 0.39$ & 0.06 & 0.07 & 0.17 & $2.35 \pm 0.13$ & $-6.17 \pm 0.38$ & 0.06 & 0.06 & 0.17 \\
\hline$r_{200}$ & $2.38 \pm 0.16$ & $-6.39 \pm 0.46$ & 0.07 & 0.09 & 0.18 & $2.29 \pm 0.15$ & $-6.06 \pm 0.41$ & 0.07 & 0.08 & 0.16 & $2.29 \pm 0.15$ & $-5.84 \pm 0.42$ & 0.07 & 0.08 & 0.17 \\
\hline \multicolumn{16}{|c|}{$L_{\mathrm{op}}-T_{\mathrm{X}}$} \\
\hline & $\alpha$ & $\beta$ & $\sigma$ & $\sigma_{T_{\mathrm{X}}}$ & $\sigma_{L_{\mathrm{op}}}$ & $\alpha$ & $\beta$ & $\sigma$ & $\sigma_{T_{\mathrm{X}}}$ & $\sigma_{L_{\mathrm{op}}}$ & $\alpha$ & $\beta$ & $\sigma$ & $\sigma_{T_{\mathrm{X}}}$ & $\sigma_{L_{\mathrm{op}}}$ \\
\hline$r_{500}$ & $1.66 \pm 0.08$ & $-0.82 \pm 0.06$ & 0.10 & 0.09 & 0.17 & $1.69 \pm 0.08$ & $-0.77 \pm 0.06$ & 0.09 & 0.08 & 0.16 & $1.65 \pm 0.08$ & $-0.50 \pm 0.06$ & 0.08 & 0.08 & 0.14 \\
\hline$r_{200}$ & $1.62 \pm 0.10$ & $-0.59 \pm 0.08$ & 0.12 & 0.13 & 0.20 & $1.64 \pm 0.09$ & $-0.53 \pm 0.07$ & 0.10 & 0.12 & 0.17 & $1.62 \pm 0.10$ & $-0.28 \pm 0.07$ & 0.09 & 0.12 & 0.16 \\
\hline
\end{tabular}


report the values of the best fit parameters plus the error at $95 \%$ confidence level and three values of the scatter: the orthogonal scatter of the relation and the scatters in both variables (in the logarithmic space). All the scatter values are expressed in dex. All the results are obtained using the mass and velocity dispersion derived from the dynamical analysis of the red members of the clusters.

\section{References}

Akritas, M. G., \& Bershady, M. A. 1996, ApJ, 470, 706

Abazajian, K., Adelman, J., Agueros, M., et al. 2003, AJ, 126, 2081 (Data Release One)

Adami, C., Mazure, A., Biviano, A., Katgert, P., \& Rhee, G. 1998, A\&A, 331, 493

Bahcall, N. A., \& Comerford, J. M. 2002, ApJ, 565, L5

Barrientos, F., Gladders, M., Yee, H., et al. 2003, Messenger, 112, 40

Beers, T. C., Geller, M. J., Huchra, J. P., et al. 1984, ApJ, 283, 33

Beers, T. C., Flynn, K., \& Gebhardt, K. 1990, AJ, 100, 32

Biviano, A., \& Girardi, M. 2003, ApJ, 585, 205

Biviano, A., \& Katgert, P. 2003, Ap\&SS, 285, 25

Biviano, A., Katgert, P., Mazure, A., et al. 1997, A\&A, 321, 84

Biviano, A., Katgert, P., Thomas, T., et al. 2002, A\&A, 387, 8

Blanton, M. R., Lupton, R. H., Maley, F. M., et al. 2003, AJ, 125, 2276 (Tiling Algorithm)

Böhringer, H., Voges, W., Huchra, J. P., et al. 2000, ApJS, 129, 435

Böhringer, H., Schuecker, P., Guzzo, L., et al. 2001, A\&A, 369, 826

Böhringer, H., Collins, C. A., Guzzo, L., et al. 2002, ApJ, 566, 93

Borgani, S., Murante, G., Springel, V., et al. 2004, MNRAS, 348, 1078

Carlberg, R. G., Yee, H. K., \& Ellingson, E. 1997a, ApJ, 478, 462

Carlberg, R. G., Yee, H. K. C., Ellingson, E., et al. 1997b, ApJ, 476, L7

den Hartog, R., \& Katgert, P. 1996, MNRAS, 279, 349

Dolag, K., Bartelmann, M., Perrotta, F., et al. 2004, A\&A, 416, 853

Donahue, M., Scharf, C. A., Mack, J., et al. 2002, ApJ, 569, 689

Eisenstein, D. J., Annis, J., Gunn, J. E., et al. 2001, AJ, 122, 2267

Ettori, S., Borgani, S., Moscardini, L., et al. 2004, MNRAS, 322

Evrard August, E., Metzler Christopher, A., \& Navarro Julio, F. 1996, ApJ, 469, 494

Fabian, A. C., Crawford, C. S., Edge, A. C., \& Mushotzky, R. F. 1994, MNRAS, 267, 779

Finoguenov, A., Reiprich, T., \& Böhringer, H. 2001, A\&A, 368, 749 (F01)

Fukugita, M., Ichikawa, T., \& Gunn, J. E. 1996, AJ, 111, 1748

Fujita, Y., \& Takahara, F. 1999, ApJ, 519, 55F

Gilbank, D. G., Bower, R. G., Castander, F. J., \& Ziegler, B. L. 2004, MNRAS, 348, 551

Girardi, M., Biviano, A., Giuricin, G., et al. 1993, ApJ, 404, 38

Girardi, M., Biviano, A., Giuricin, G., et al. 1995, ApJ, 438, 527

Girardi, M., Giuricin, G., Mardirossian, F., Mezzetti, M., et al. 1998, ApJ, 505, 74

Girardi, M., Borgani, S., Giuricin, G., et al. 2000, ApJ, 530, 62

Girardi, M., Manzato, P., Mezzetti, M., et al. 2002, ApJ, 569, 720
Gunn, J. E., Carr, M. A., Rockosi, C. M., et al. 1998, AJ, 116, 3040 (SDSS Camera)

den Hartog, R., \& Katgert, P. 1996, MNRAS, 279, 349

Hogg, D.W., Finkbeiner, D. P., Schlegel, D. J., \& Gunn, J. E. 2001, AJ, 122, 2129

Horner, D. 2001, Ph.D. Thesis, University of Maryland

Jones, C., \& Forman, W. 1984, ApJ, 276, 38

Katgert, P., Biviano, A., \& Mazure, A. 2004, ApJ, 600, 657

Kochanek, C. S., White, M., Huchra, J., et al. 2003, ApJ, 585, 161

Lin, Y.-T., Mohr, J. J., \& Stanford, S. A. 2003, ApJ, 591, 749

Lin, Y.-T., Mohr, J. J., \& Stanford, S. A. 2004, ApJ, 610, 745

Lupton, R. H., Gunn, J. E., \& Szalay, A. S. 1999, AJ, 118, 1406

Lupton, R., Gunn, J. E., Ivezić, Z., et al. 2001, in Astronomical Data Analysis Software and Systems X, ed. F. R. Harnden, Jr., F. A. Primini, \& H. E. Payne (San Francisco: ASP), ASP Conf. Ser., 238, 269 [arXiv: astro-ph/0101420]

Markevitch, M. 1998, ApJ, 504, 27 (M98)

Moss, C., \& Dickens, R. J. 1977, MNRAS, 178, 701

Mulchaey, J. S., Davis, D. S., Mushotzky, R. F., \& Burstein, D. 2003, ApJS, 145, 39

Navarro, J. F., Frenk, C. S., \& White, S. D. M. 1996, ApJ, 462, 563

Navarro, J. F., Frenk, C. S., \& White, S. D. M. 1997, ApJ, 490, 493

Oort, J. H. 1958, in La structure et l'evolution de l'univers, ed. R. Stoopes (Brussels: Solvay Inst.), 163

Ortiz-Gil, A., Guzzo, L., Schücker, P., et al. 2004, MNRAS, 348, 325

Ota, N., \& Mitsuda, K. 2002, ApJ, 567, L23

Popesso, P., Böhringer, H., Brinkmann, J., et al. 2004, A\&A, 423, 449

Ramella, M., Boschin, W., Geller, M. J., Mahdavi, A., \& Rines, K. 2004, AJ, in press [arXiv: astro-ph/0407640]

Reiprich, T., \& Böhringer, H. 2002, ApJ, 567, 740 (R02)

Rines, K., Geller, M. J., Diaferio, A., et al. 2004, AJ, 128, 1078

Rines, K., Geller, M. J., Kurtz, M. J., \& Diaferio, A. 2003, AJ, 126, 2152

Retzlaff, J. 2001, XXIst Moriond Astrophysics Meeting, March 10-17, 2001 Savoie, France ed. D. M. Neumann, \& J. T. T. Van

Rines, K., Geller, M. J., Diaferio, A., Kurtz, M. J., \& Jarrett, T. H. 2004, AJ, 128, 1078

Schaeffer, R., Maurogordato, S., Cappi, A., et al. 1993, MNRAS, 263L, 21S

Smail, I., Ellis, R. S., Dressler, A., et al. 1997, ApJ, 479, 70 S

Smith, S. 1936, ApJ, 83, 23S

Smith, J. A., Tucker, D. L., Kent, S. M., et al. 2002, AJ, 123, 2121

Stoughton, C., Lupton, R. H., Bernardi, M., et al. 2002, AJ, 123, 485

Strauss, M. A., Weinberg, D. H., Lupton, R. H., et al. 2002, AJ, 124, 1810

The, L. S., \& White, S. D. M. 1986, AJ, 92, 1248

Tegmark, M., Strauss, M., Blanton, M., et al. 2004, PhRvD

Voges, W., Aschenbach, B., Boller, T., et al. 1999, A\&A, 349, 389

Wu, X.-P. 2000, MNRAS, 316, 299

Yasuda, N., Fukugita, M., Narayanan, V. K., et al. 2001, AJ, 122, 1104

Yee, H. K. C., \& Ellingson, E. 2003, ApJ, 585, 226

York, D. G., Adelman, J., Anderson, J. E., et al. 2000, AJ, 120, 1579

Zwicky, F. 1933, Helv. Phys. Acta, 6, 110

Zwicky, F. 1937, ApJ, 86, 127 